\title{
WHEN THUE-MORSE MEETS KOCH
}

\author{
JUN MA AND JUDY HOLDENER
}

\begin{abstract}
In this paper, we reveal a remarkable connection between the Thue-Morse sequence and the Koch snowflake. Using turtle geometry and polygon maps, we realize the Thue-Morse sequence as the limit of polygonal curves in the plane. We then prove that a sequence of such curves converges to the Koch snowflake in the Hausdorff metric. In the final section we consider generalized Thue-Morse sequences and provide a characterization of those that encode curves converging to the Koch snowflake.
\end{abstract}

\section{INTRODUCTION}

The Thue-Morse sequence and the Koch snowflake have much in common. Both are defined iteratively. Both exhibit properties of self-similarity. Both first appeared in the early 1900's (the Koch snowflake in 1906 and the Thue-Morse sequence in 1912). And both continue to appear frequently - yet independently - in popular mathematical writing today. In this paper, we will show that the commonality between these two famous objects is deeper yet. Indeed, by realizing the ThueMorse sequence geometrically as the limit of polygonal curves in the plane, we will show that the connection between the Thue-Morse sequence and the Koch snowflake is much stronger than one might expect.

The Thue-Morse sequence is a two symbol sequence typically defined by iterating a substitution map $\sigma$. Given the alphabet $A=\{a, b\}$, define the morphism $\sigma: \mathcal{A}^{*} \rightarrow$ $\mathcal{A}^{*}$ by setting $\sigma(a)=a b$ and $\sigma(b)=b a$. If $\sigma^{0}=a$, we see that $\sigma$ generates the sequence of words:

$$
\left\{\sigma^{n}(a)\right\}_{n \geq 0}=a, a b, a b b a, a b b a b a a b, a b b a b a a b b a a b a b b a, \ldots
$$

This sequence converges to what is commonly known as the Thue-Morse sequence:

$$
t=\lim _{n \rightarrow \infty} \sigma^{n}(a)=a b b a b a a b b a a b a b b a b a a b a b b a a b b a b a a b \ldots
$$

Of course, there is nothing special about the symbols $a$ and $b$, and in this article we choose to use the alphabet $\Sigma=\{F, L\}$, where $F$ and $L$ represent commands for a turtle in the plane (in the sense of the turtle geometry developed in the early 1980's [1].) The symbol $F$ represents a forward motion of the turtle in the plane by one unit and $L$ a counterclockwise rotation of the turtle by the fixed angle $\theta=\pi / 3$.

Definition 1.0.1. Let $\Sigma=\{F, L\}$. Then the Thue-Morse turtle programs of degree $n$, denoted by $T M_{n}$ and $\overline{T M}_{n}$, are defined to be the following words in $\Sigma^{*}$

$$
T M_{n}=\sigma^{n}(F) \text { and } \overline{T M}_{n}=\sigma^{n}(L)
$$

1991 Mathematics Subject Classification. Primary 68R15; Secondary 28A80.

Key words and phrases. Thue-Morse sequence, Koch snowflake, recurrent curves. 
The trajectories encoded by the Thue-Morse turtle programs turn out to be surprisingly interesting. Figures 1 and 2 below show the results of the Thue-Morse turtle programs of degrees 4 through 10 .

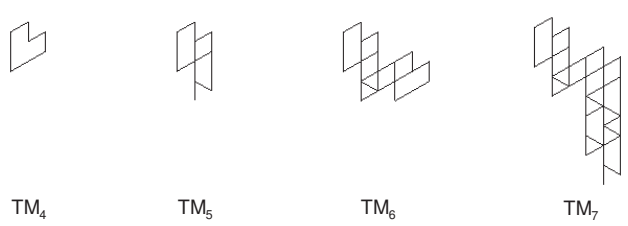

Figure 1. Thue-Morse turtle programs of degrees 4 through 7

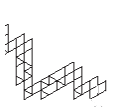

$\mathrm{TM}_{8}$

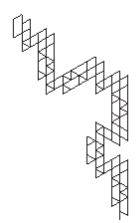

$\mathrm{TM}_{9}$

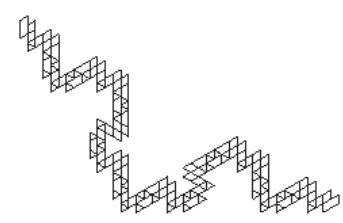

$\mathrm{TM}_{10}$

Figure 2. Thue-Morse turtle programs of degrees 8 through 10

Indeed, the trajectories corresponding to the even terms of the Thue-Morse sequence are starting to resemble the familiar Koch snowflake! Skeptical? Consider $T M_{14}$.

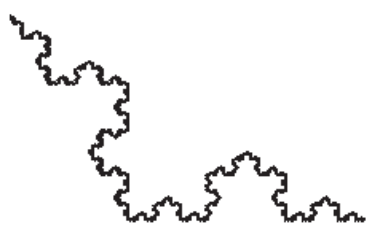

Figure 3. The Thue-Morse turtle program of degree 14

The primary purpose of this paper is to examine the sequence of curves encoded by Thue-Morse turtle programs $\left\{T M_{2 n}\right\}$. In subsections 3.1-3.3, we use turtle geometry and polygon maps to realize the programs $T M_{2 n}$ as polygonal curves in the plane. We then present a few useful preliminary results involving computations in the Hausdorff metric (subsection 3.4 and 3.5). In our two main results Convergence Theorems I and II - we prove that a sequence of Thue-Morse turtle trajectories does, in fact, converge to the Koch Snowflake. In the final section, we consider possible generalizations of Thue-Morse turtle programs, exploring the existence of other iterative turtle programs that produce curves converging to the Koch snowflake. 


\section{Two Famous Mathematical OBjects}

2.1. The Thue-Morse Sequence. The Norwegian mathematician Axel Thue first discovered the Thue-Morse sequence in 1912 while studying avoidable patterns in sequences of symbols [12]. Thue proved that the sequence is overlapfree, meaning that it contains no substrings of the form wuwuw.) The sequence was later rediscovered by Marston Morse (1917), who was interested in the aperiodicity of the sequence [8]. Since the early 1900's, interest in the Thue-Morse sequence has continued. In 1944, Morse and Hedlund proved that the sequence is cubefree (meaning it contains no substrings of the form $w w w$ ) [9], and there have been numerous writings of late that focus on the many interesting properties exhibited by the sequence $[[3],[7],[6],[10]]$. Below we list a few basic properties that are relevant to this paper. The properties, as stated, involve even terms only because it is the even terms of the Thue-Morse sequence that appear to be converging to the Koch snowflake.

Property 2.1.1. For all $n \in \mathbb{N}, T M_{2 n+2}=T M_{2 n} \overline{T M}_{2 n} \overline{T M}_{2 n} T M_{2 n}$ and $\overline{T M}_{2 n+2}=$ $\overline{T M}_{2 n} T M_{2 n} T M_{2 n} \overline{T M}_{2 n}$.

Note that Property 2.1.1 implies that the even terms of $\left\{T M_{n}\right\}$ are palindromes. This ensures the bilateral symmetry of the polygonal curves they encode.

Property 2.1.2. Given $\Omega_{2 k}=\left\{T M_{2 k}, \overline{T M}_{2 k}\right\}$ and $n \geq k$,

$$
T M_{2 n}=\sigma^{2 n-2 k}\left(T M_{2 k}\right) \in \Omega_{2 k}^{*} \text { and } \overline{T M}_{2 n}=\sigma^{2 n-2 k}\left(\overline{T M}_{2 k}\right) \in \Omega_{2 k}^{*}
$$

Property 2.1.3. If $\tau: \Sigma^{*} \rightarrow \Sigma^{*}$ is the morphism defined by $\tau(F)=L$ and $\tau(L)=F$, then $\tau\left(T M_{2 k}\right)=\overline{T M}_{2 k}$ and $\tau\left(\overline{T M}_{2 k}\right)=T M_{2 k}$.

Remark 2.1.4. The bar notation, $\overline{T M}_{2 n} \equiv \sigma^{2 n}(L)$, was actually chosen to reflect Property 2.1.3. In general, if $w$ is a word over a two symbol alphabet $\{a, b\}$, then $\bar{w}$ denotes the word obtained by exchanging all $a$ 's to $b$ 's and all $b$ 's to $a$ 's [3].

2.2. The Koch Snowflake. Now considered a classical fractal object, the Koch snowflake was first introduced by Helge von Koch in 1906 [13]. It is constructed by starting with a line segment of unit length, extracting the middle third and replacing it with two line segments of length $1 / 3$ (see Figure 4 ). The process is continued indefinitely, with the middle third of any line segment at each stage being replaced with two line segments of length equal to $1 / 3$ of the line segment.

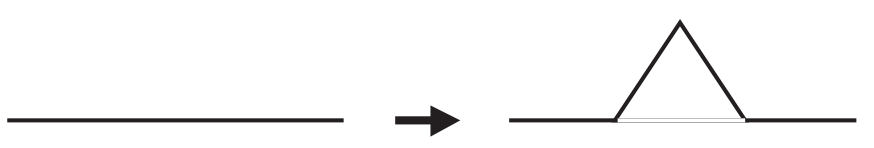

Figure 4. Edge replacement in the Koch snowflake

The Koch snowflake is commonly defined by way of a Lindenmayer system with initial string $F$ and rewriting rule $F \rightarrow F-F++F-F$, where "+" denotes a counterclockwise rotation of $\pi / 3$ radians and "-." a clockwise rotation by the same amount [11]. The "reflected" rewriting rule $F \rightarrow F+F--F+F$ amounts to the morphism $\kappa: \Sigma^{*} \rightarrow \Sigma^{*}$ defined by $\kappa(F)=F L^{5} F L^{2} F L^{5} F$ and $\kappa(L)=L$, where $\Sigma^{*}$ is the monoid of words over $\Sigma$ (the operation being concatenation.) Note that $F L^{5} F L^{2} F L^{5} F$ can be factored in two ways: 
(1) $\left(F L^{4}\right)(L F L)(L F L)\left(L^{4} F\right)$

(2) $(F L)\left(L^{4} F L\right)\left(L F L^{4}\right)(L F)$

Defining $a=L^{4} F L^{4}$ and $b=L F L$ we see that the rewriting rule $\kappa$ is reminiscent of $\sigma^{2}$, which generates the even terms of the Thue-Morse sequence (in particular, see Property 2.1.1). For if we assume that $L^{6}$ is the empty word (representing a counterclockwise rotation of $2 \pi$ ), then

$$
\begin{aligned}
\kappa(a)=\kappa\left(L^{4} F L^{4}\right)=L^{4} \kappa(F) L^{4} & =L^{4}\left(F L^{4}\right)(L F L)(L F L)\left(L^{4} F\right) L^{4} \\
& =a b b a=\sigma^{2}(a)
\end{aligned}
$$

and

$$
\begin{aligned}
\kappa(b)=\kappa(L F L)=L \kappa(F) L & =L(F L)\left(L^{4} F L\right)\left(L F L^{4}\right)(L F) L \\
& =L(F L)\left(L^{4} F L\right) L^{6}\left(L F L^{4}\right)(L F) L \\
& =b a a b=\sigma^{2}(b)
\end{aligned}
$$

Moreover, further iteration of $\kappa$ will result in the following.

Proposition 2.2.1. Let $n \in \mathbb{N}$, and consider the set of all words over $\Sigma$ subject to the relation $L^{6}=\varepsilon$ (where $\varepsilon$ is the empty word). Then the following two identities hold true.

(1) $\kappa^{n}\left(L^{4} F L^{4}\right)=\sigma^{2 n}\left(L^{4} F L^{4}\right)$

(2) $\kappa^{n}(L F L)=\sigma^{2 n}(L F L)$

Proof. Proceed by induction. Clearly, $\kappa^{0}\left(L^{4} F L^{4}\right)=L^{4} F L^{4}=\sigma^{0}\left(L^{4} F L^{4}\right)$ and $\kappa^{0}(L F L)=L F L=\sigma^{0}(L F L)$. Now suppose that (1) and (2) hold for $n$ and compute:

$$
\begin{aligned}
\sigma^{2 n+2}\left(L^{4} F L^{4}\right) & =\sigma^{2 n}\left(L^{4} F L^{4}\right) \sigma^{2 n}(L F L) \sigma^{2 n}(L F L) \sigma^{2 n}\left(L^{4} F L^{4}\right) \\
& =L^{4} \kappa^{n}(F) L^{4} L \kappa^{n}(F) L L \kappa^{n}(F) L L^{4} \kappa^{n}(F) L^{4} \\
& =L^{4}\left[\kappa^{n}(F) L^{5} \kappa^{n}(F) L^{2} \kappa^{n}(F) L^{5} \kappa^{n}(F)\right] L^{4} \\
& =\kappa^{n+1}\left(L^{4} F L^{4}\right) \\
\sigma^{2 n+2}(L F L) & =\sigma^{2 n}(L F L) \sigma^{2 n}\left(L^{4} F L^{4}\right) \sigma^{2 n}\left(L^{4} F L^{4}\right) \sigma^{2 n}(L F L) \\
& =L \kappa^{n}(F) L L^{4} \kappa^{n}(F) L^{4} L^{4} \kappa^{n}(F) L^{4} L \kappa^{n}(F) L \\
& =L\left[\kappa^{n}(F) L^{5} \kappa^{n}(F) L^{2} \kappa^{n}(F) L^{5} \kappa^{n}(F)\right] L \\
& =\kappa^{n+1}(L F L)
\end{aligned}
$$

Finally, we note that the trajectories encoded by $\kappa\left(L^{4} F L^{4}\right)$ and $\kappa(L F L)$ are simply rotations of that encoded by $\kappa(F)$. Hence $\kappa\left(L^{4} F L^{4}\right)$ provides another encoding of the Koch snowflake. In light of the previous proposition, this means that the sequence $\left\{\sigma^{2 n}\left(L^{4} F L^{4}\right)\right\}_{n \geq 1}$ also encodes the Koch snowflake, and it is this formulation that we will be using in this paper. Moreover, we can replace $F$ in the rewriting rule with any other turtle sequence that restores the initial heading. For example, any power of $F$ would work.

\section{Preliminaries}

3.1. Turtle Geometry and the Position Homomorphism. A turtle program is defined to be any word over the alphabet $\Sigma=\{L, F\}$ where $F$ denotes a forward motion of the turtle by one unit and $L$ a counterclockwise rotation by some fixed angle $\theta$. If $\theta=2 \pi / N$, then the set of all words over $\Sigma$ subject to the relation $\mathcal{R}=\left\{L^{N}=\varepsilon\right\}$ is denoted by $\Sigma_{\mathcal{R}}^{*}$. The length of a turtle program, $|w|$, is the 
number of letters in $w$. By $|w|_{L}$, we denote the number of $L$ 's in $w$, and similarly, $|w|_{F}$ denotes the number of $F$ 's.

A turtle state is an ordered pair $(\vec{r}, \hat{v})$ consisting of a position vector $\vec{r} \in \mathbb{R}^{2}$, and a unit vector $\hat{v}$ describing the turtle's heading. The basic turtle commands $F$ and $L$ define certain changes in the turtle's state; the command $F$ represents the transformation $T_{F}$ mapping the state $(\vec{r}, \hat{v})$ to the state $(\vec{r}+\hat{v}, \hat{v})$, and $L$ represents the transformation $T_{L}$ mapping $(\vec{r}, \hat{v})$ to $\left(\vec{r}, R_{\theta} \hat{v}\right)$, where $R_{\theta}$ is the rotation matrix

$$
R_{\theta}=\left[\begin{array}{rr}
\cos \theta & -\sin \theta \\
\sin \theta & \cos \theta
\end{array}\right], \theta=2 \pi / N
$$

For the purposes of this paper, $\theta$ will always be fixed at $\pi / 3$ (and hence $\mathcal{R}=\left\{L^{6}=\right.$ $\varepsilon\}$.

A string $w$ of $F$ 's and $L$ 's then describes the general turtle transformation $T_{w}$ consisting of compositions of these two basic transformations. Such transformations $T_{w}$ can be expressed in the form $T_{w}(\vec{r}, \hat{v})=(\vec{r}+M \hat{v}, R \hat{v})$, where $M$ is a matrix of the form $\left[\begin{array}{rr}a & -b \\ b & a\end{array}\right] \in M_{2}(\mathbb{R})$ and $R=R_{\theta}^{k}$ for some positive integer $k$. As described in [5], the set of pairs $(M, R) \in \mathcal{G}=M_{2}(\mathbb{R}) \times\left\langle R_{\theta}\right\rangle$ is a group under the binary operation

$$
\left(M_{1}, R_{1}\right)\left(M_{2}, R_{2}\right)=\left(M_{1}+R_{1} M_{2}, R_{1} R_{2}\right),
$$

and there is a homomorphism $\psi: \Sigma_{\mathcal{R}}^{*} \rightarrow \mathcal{G}$ defined by

$$
\psi(w)=\left(\sum_{j=1}^{k} m_{j} R_{\theta}^{\sum_{i=1}^{j} n_{i}}, R_{\theta}^{\sum_{i=1}^{k} n_{i}}\right)
$$

for any $w=\prod_{i=1}^{k} L^{n_{i}} F^{m_{i}} \in \Sigma_{\mathcal{R}}^{*}$. The map $\psi$ is useful, because it allows us to compute the turtle's position and heading with ease. Indeed, if the turtle starts with the state $(\vec{r}, \hat{v})=(\langle 0,0\rangle,\langle 0,1\rangle)$ and follows the string of commands $w$, then its new position and heading are $\pi_{1} \circ \psi(w) \cdot \hat{v}$ and $\pi_{2} \circ \psi(w) \cdot \hat{v}$, respectively. In this paper, we define the position homomorphism $g: \Sigma_{\mathcal{R}}^{*} \rightarrow \mathbb{R}^{2}$ as follows, with the assumption that the turtle's initial state is always $(\vec{r}, \hat{v})=(\langle 0,0\rangle,\langle 0,1\rangle)$.

Definition 3.1.1. Given $\hat{v}_{0}=\langle 0,1\rangle$ and the map $\phi_{0}: M_{2}(\mathbb{R}) \rightarrow \mathbb{R}^{2}$ defined by $\phi_{0}(M)=M \cdot \hat{v}_{0}$, the position homomorphism $g: \Sigma_{\mathcal{R}}^{*} \rightarrow \mathbb{R}^{2}$ is defined to be the composition $g=\phi_{0} \circ \pi_{1} \circ \psi$.

$$
g: \Sigma_{\mathcal{R}}^{*} \stackrel{\psi}{\rightarrow} M_{2}(\mathbb{R}) \times\left\langle R_{\theta}\right\rangle \stackrel{\pi_{1}}{\rightarrow} M_{2}(\mathbb{R}) \stackrel{\phi_{0}}{\rightarrow} \mathbb{R}^{2}
$$

It is worth noting that although the position homomorphism furnishes the final position of the turtle only, it does retain some information about the turtle's heading. In particular, if $w=w_{1} w_{2}$, and $\psi\left(w_{1}\right)=\left(M_{1}, R_{1}\right), \psi\left(w_{2}\right)=\left(M_{2}, R_{2}\right)$, then

$$
\begin{aligned}
g\left(w_{1} w_{2}\right) & =\pi_{1} \circ \psi\left(w_{1} w_{2}\right) \cdot \hat{v}_{0} \\
& =\left(M_{1}+R_{1} \cdot M_{2}\right) \cdot \hat{v}_{0} \\
& =M_{1} \cdot \hat{v}_{0}+R_{1} \cdot M_{2} \cdot \hat{v}_{0} \\
& =g\left(w_{1}\right)+R_{1} \cdot g\left(w_{2}\right),
\end{aligned}
$$

and we see that $\psi\left(w_{1} w_{2}\right)$ depends on $R_{1}=\pi_{2} \circ \psi\left(w_{1}\right)$. Furthermore, by defining the multiplication on $G=\left\{g(w) \mid w \in \Sigma_{\mathcal{R}}^{*}\right\}$ to be $g\left(w_{1}\right) \boxplus g\left(w_{2}\right)=g\left(w_{1}\right)+\pi_{2} \circ$ 
$\psi\left(w_{1}\right) \cdot g\left(w_{2}\right)$, we are simply equipping $G$ with the multiplication it has inherited from $\mathcal{G}=M_{2}(R) \times\left\langle R_{\theta}\right\rangle$. The following proposition is easy to check, so we will state it without proof.

Proposition 3.1.2. The set $G=\left\{g(w) \mid w \in \Sigma_{\mathcal{R}}^{*}\right\}$ is a group under the binary operation

$$
g\left(w_{1}\right) \boxplus g\left(w_{2}\right)=g\left(w_{1}\right)+\pi_{2} \circ \psi\left(w_{1}\right) \cdot g\left(w_{2}\right)
$$

and $g: \Sigma_{\mathcal{R}}^{*} \rightarrow \mathbb{R}^{2}$ is a homomorphism.

3.2. Thue-Morse Turtle Programs. The goal of the current section is to compute the heading and position of a turtle that has followed the Thue-Morse turtle programs $T M_{2 n}$ and $\overline{T M}_{2 n}$. The next lemma will be helpful.

Lemma 3.2.1. Let $w, w^{\prime} \in \Sigma_{\mathcal{R}}^{*}$ be two words satisfying $\pi_{1} \circ \psi(w)=M, \pi_{1} \circ \psi\left(w^{\prime}\right)=$ $M^{\prime}$ and $\pi_{2} \circ \psi(w)=\pi_{2} \circ \psi\left(w^{\prime}\right)=R_{\theta}^{2}$. Then for all integers $n \geq 0$,

(1) $\pi_{2} \circ \psi\left(\sigma^{2 n}(w)\right)=\pi_{2} \circ \psi\left(\sigma^{2 n}\left(w^{\prime}\right)\right)=R_{\theta}^{2}$.

(2) $\pi_{1} \circ \psi\left(\sigma^{2 n}(w)\right)=\frac{3^{n}+1}{2} \cdot M-\frac{3^{n}-1}{2} \cdot M^{\prime}$ and $\pi_{1} \circ \psi\left(\sigma^{2 n}\left(w^{\prime}\right)\right)=\frac{3^{n}+1}{2} \cdot M^{\prime}-\frac{3^{n}-1}{2} \cdot M$

Proof. We prove (1) by induction. Assuming $\pi_{2} \circ \psi(w)=\pi_{2} \circ \psi\left(w^{\prime}\right)=R_{\theta}^{2}$, suppose $\pi_{2} \circ \psi\left(\sigma^{2 n}(w)\right)=\pi_{2} \circ \psi\left(\sigma^{2 n}\left(w^{\prime}\right)\right)=R_{\theta}^{2}$. Then

$$
\begin{aligned}
\pi_{2} \circ \psi\left(\sigma^{2 n+2}(w)\right) & =\pi_{2} \circ \psi\left(\sigma^{2 n}(w) \sigma^{2 n}\left(w^{\prime}\right) \sigma^{2 n}\left(w^{\prime}\right) \sigma^{2 n}(w)\right) \\
& =\pi_{2} \circ \psi\left(\sigma^{2 n}(w)\right) \cdot\left(\pi_{2} \circ \psi\left(\sigma^{2 n}\left(w^{\prime}\right)\right)^{2} \cdot \pi_{2} \circ \psi\left(\sigma^{2 n}(w)\right)\right. \\
& =\left(R_{\theta}^{2}\right)^{4} \\
& =R_{\theta}^{2}
\end{aligned}
$$

To prove (2), apply induction once again. The base case $(\mathrm{n}=0)$ follows from the assumption that $\pi_{1} \circ \psi(w)=M$ and $\pi_{1} \circ \psi\left(w^{\prime}\right)=M^{\prime}$. Setting $M_{n}=\pi_{1} \circ \psi\left(\sigma^{2 n}(w)\right)$ and $M_{n}^{\prime}=\pi_{1} \circ \psi\left(\sigma^{2 n}\left(w^{\prime}\right)\right)$ and recalling that $\theta=\pi / 3$ so that $R_{\theta}^{6}=I$ and $R_{\theta}^{2}+R_{\theta}^{4}=$ $-I$, we compute:

$$
\begin{aligned}
\pi_{1} \circ \psi\left(\sigma^{2 n+2}(w)\right) & =\pi_{1} \circ \psi\left(\sigma^{2 n}(w) \sigma^{2 n}\left(w^{\prime}\right) \sigma^{2 n}\left(w^{\prime}\right) \sigma^{2 n}(w)\right) \\
& =M_{n}+R_{\theta}^{2} \cdot M_{n}^{\prime}+R_{\theta}^{4} \cdot M_{n}^{\prime}+R_{\theta}^{6} \cdot M_{n} \\
& =\left(I+R_{\theta}^{6}\right) \cdot M_{n}+\left(R_{\theta}^{2}+R_{\theta}^{4}\right) \cdot M_{n}^{\prime} \\
& =2 M_{n}-M_{n}^{\prime}
\end{aligned}
$$

Assuming $M_{n}=\pi_{1} \circ \psi\left(\sigma^{2 n}(w)\right)=\frac{3^{n}+1}{2} \cdot M-\frac{3^{n}-1}{2} \cdot M^{\prime}$, the above computation then yields the desired result.

With the previous lemma in hand, we are now ready to compute the exact position and heading resulting from a Thue-Morse turtle program.

Proposition 3.2.2. For all $n \in \mathbb{N}$,

(1) $\pi_{2} \circ \psi\left(T M_{2 n}\right)=\pi_{2} \circ \psi\left(\overline{T M}_{2 n}\right)=R_{\theta}^{2}$

(2) $\pi_{1} \circ \psi\left(T M_{2 n}\right)=\frac{3-3^{n-1}}{2} \cdot R_{\theta}$

(3) $\pi_{1} \circ \psi\left(\overline{T M}_{2 n}\right)=\frac{3+3^{n-1}}{2} \cdot R_{\theta}$

Proof. Let $w=T M_{2}=F L L F$ and $w^{\prime}=\overline{T M_{2}}=L F F L$. Since $\pi_{2} \circ \psi\left(T M_{2}\right)=$ $\pi_{2} \circ \psi\left(\overline{T M_{2}}\right)=R_{\theta}^{2}$, (1) follows directly from (1) of the previous lemma. 
The proof of (2) follows from the following computation. Let $M=\pi_{1} \circ \psi\left(T M_{2}\right)=$ $R_{\theta}$ and $M^{\prime}=\pi_{1} \circ \psi\left(\overline{T M}_{2}\right)=2 R_{\theta}$

$$
\begin{aligned}
\pi_{1} \circ \psi\left(T M_{2 n}\right) & =\pi_{1} \circ \psi\left(\sigma^{2 n-2}\left(T M_{2}\right)\right) \\
& =\frac{3^{n-1}+1}{2} \cdot R_{\theta}-\frac{3^{n-1}-1}{2} \cdot 2 R_{\theta} \\
& =\frac{3-3^{n-1}}{2} \cdot R_{\theta}
\end{aligned}
$$

The proof of (3) is similar. Let $M=\pi_{1} \circ \psi\left(\overline{T M}_{2}\right)=2 R_{\theta}$ and $M^{\prime}=\pi_{1} \circ \psi\left(T M_{2}\right)=$ $R_{\theta}$.

$$
\begin{aligned}
\pi_{1} \circ \psi\left(\overline{T M}_{2 n}\right) & =\pi_{1} \circ \psi\left(\sigma^{2 n-2}\left(\overline{T M}_{2}\right)\right) \\
& =\frac{3^{n-1}+1}{2} \cdot 2 R_{\theta}-\frac{3^{n-1}-1}{2} \cdot R_{\theta} \\
& =\frac{3+3^{n-1}}{2} \cdot R_{\theta}
\end{aligned}
$$

We conclude this subsection with two more definitions that will prove to be helpful in the proofs of the main convergence theorems.

Definition 3.2.3. For integers $n \geq 3$ the length of $g\left(\overrightarrow{T M_{2 n}}\right)$, denoted by $l_{n}$, is defined to be $l_{n}=\left|\overrightarrow{g\left(T M_{2 n}\right)}\right|$.

Recalling that we set the initial position vector of the turtle to be $\overrightarrow{r_{0}}=\langle 0,0\rangle$, we can use Proposition 3.2.2 to compute $l_{n}$ exactly.

$$
\begin{aligned}
l_{n} & =\left|\overrightarrow{g\left(T M_{2 n}\right)}\right| \\
& =\left|\overrightarrow{r_{0}}+\frac{3-3^{n-1}}{2} \cdot R_{\theta} \cdot \overrightarrow{v_{0}}\right| \\
& =\left|\frac{3-3^{n-1}}{2} \cdot R_{\theta} \cdot \overrightarrow{v_{0}}\right| \\
& =3^{n-1}-3
\end{aligned}
$$

For the reader wondering why we define $l_{n}$ for $n \geq 3$ only, note that it is precisely the Thue-Morse turtle program of order 3 (that is, $T M_{6}$ ) that corresponds to what is commonly considered to be the initial configuration of the Koch Snowflake (see Figure 1). Hence it makes sense to consider $T M_{6}$ as our starting point. With this in mind, we then define the scaling factor $S_{n}$ of each iteration $T M_{n}$ as follows.

Definition 3.2.4. For $n \geq 3$, the scaling factor $S_{n}$ of $T M_{2 n}$ is defined to be

$$
\begin{aligned}
S_{n} & =l_{3} / l_{n} \\
& =3 / l_{n} \\
& =\frac{2}{3^{n-2}-1}
\end{aligned}
$$

3.3. The Polygon Map. Our next goal is to realize turtle programs (in particular Thue-Morse turtle programs) as polygonal curves in the plane. Let $S$ be a subset of $\Sigma_{\mathcal{R}}^{*}$ and let $\mathcal{H}\left(\mathbb{R}^{2}\right)$ be the set of nonempty compact subsets of $\mathbb{R}^{2}$. Equipped with the homomorphism $g: \Sigma_{\mathcal{R}}^{*} \rightarrow \mathbb{R}^{2}$, we can then define a map $K[\cdot]: S \rightarrow \mathcal{H}\left(\mathbb{R}^{2}\right)$ that assigns a polygon to each word over $S$. As F. M. Dekking presents in [[4], p. 80], define the polygon map $K[\cdot]$ on $s \in S$ to be $K[s]=\{\alpha \cdot g(s): 0 \leq \alpha \leq 1\}$, and extend the map to all words over $S$ by requiring that $K[V W]=K[V] \cup(g(V) \boxplus K[W])$ for any $V, W \in S^{*}$ where $g(V) \boxplus K[W]:=g(V)+\pi_{2} \circ \psi(V) \cdot K[W]$.

As indicated above, there can be many different ways of realizing a turtle program in the plane. As the examples below illustrate, the realization will depend on the way we define $S$. 
Example 3.3.1. The most general polygon map is obtained by defining $S=\Sigma^{*}$. In this case, $K_{\text {turt }}: \Sigma^{*} \rightarrow \mathbb{R}^{2}$ is the map one typically associates with turtle geometry. That is, $K_{\text {turt }}: \Sigma^{*} \rightarrow \mathbb{R}^{2}$ assigns to each word $w=a_{1} a_{2} a_{3} \ldots a_{k} \in \Sigma^{*}$ the trajectory traversed by a turtle that follows each command $a_{i} \in\{F, L\}$, in turn, starting with $a_{1}$ and finishing with $a_{n}$.

Example 3.3.2. Let $\Lambda=\left\{L^{4} F^{3} L^{4}, L F^{3} L\right\}$, and consider the polygon map $K_{k s}$ : $\Lambda^{*} \rightarrow \mathcal{H}\left(\mathbb{R}^{2}\right)$. In light of Proposition 2.2.1, the polygon $K_{k s}\left[\sigma^{2 n}\left(L^{4} F^{3} L^{4}\right)\right]$ is the $n^{\text {th }}$ stage of the Koch snowflake. Defining $K S_{2 n} \equiv \sigma^{2 n}\left(L^{4} F^{3} L^{4}\right)$ and $\mathcal{K}_{2 n}=$ $\frac{1}{3^{n}} K_{k s}\left[K S_{2 n}\right]$, the Koch snowflake $\mathcal{K}$ can then be defined as the limit: $\lim _{n \rightarrow \infty} \mathcal{K}_{2 n}$. Here the limit is taken over nonempty compact subsets of $\mathbb{R}^{2}$ under the Hausdorff metric (see the next section).

Example 3.3.3. For all integers $k \geq 0$, if $\Omega_{2 k}=\left\{T M_{2 k}, \overline{T M}_{2 k}\right\}$ then the polygon maps $\left\{K_{2 k}: \Omega^{*} \rightarrow \mathcal{H}\left(\mathbb{R}^{2}\right)\right\}$ produce polygonal curves constructed out of the basic component edges $K_{2 k}\left[T M_{2 k}\right]$ and $K_{2 k}\left[\overline{T M}_{2 k}\right]$. For example, the polygons $K_{6}\left[T M_{10}\right]$ and $K_{8}\left[T M_{10}\right]$ are depicted in Figure 5 below. These polygon maps will play a critical role in the proofs of the convergence theorems.
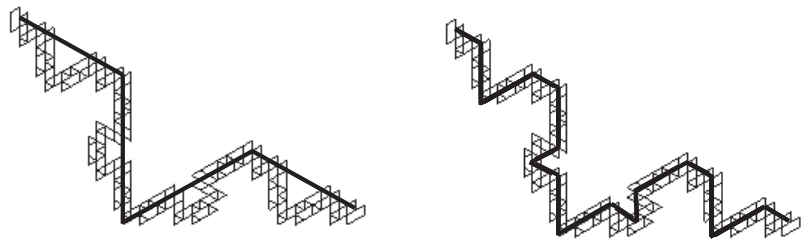

FiguRE 5. Left: $K_{8}\left[T M_{10}\right]$ overlaying $K_{0}\left[T M_{10}\right]=K_{\text {turt }}\left[T M_{10}\right]$ Right: $K_{6}\left[T M_{10}\right]$ overlaying $K_{0}\left[T M_{10}\right]=K_{\text {turt }}\left[T M_{10}\right]$

3.4. The Hausdorff Metric. The distance between two subsets of a metric space is defined using the Hausdorff metric (see [2]). Given the complete metric space $\mathbb{R}^{2}$ under the Euclidean metric $d$ and $\mathcal{H}\left(\mathbb{R}^{2}\right)$, the space of nonempty compact subsets of $\mathbb{R}^{2}$, the Hausdorff distance between two points $A, B \in \mathcal{H}\left(\mathbb{R}^{2}\right)$ is defined by

$$
h(A, B)=\max \{d(A, B), d(B, A)\},
$$

where $d(A, B)$ is the Euclidean distance between two sets:

$$
d(A, B)=\max \{d(x, B): x \in A\} .
$$

Introducing the notation $d(A, B) \vee d(B, A)$ to denote $\max \{d(A, B), d(B, A)\}$, we get the following definition.

Definition 3.4.1. The Hausdorff distance between two sets $A, B \in \mathcal{H}\left(\mathbb{R}^{2}\right)$ is defined to be $h(A, B)=d(A, B) \vee d(B, A)$.

A particularly simple situation is the case where the two sets $A$ and $B$ are parallel line segments. As Lemma 3.4.2 below indicates, in this case the Hausdorf distance $h(A, B)$ is computed easily using the (Euclidean) distances between the endpoints.

Lemma 3.4.2. If $\overline{A B}$ and $\overline{C D}$ are two parallel line segments in $\mathbb{R}^{2}$ with $\overrightarrow{A B}$ and $\overrightarrow{C D}$ pointing towards the same direction, then $h(\overline{A B}, \overline{C D})=d(A, C) \vee d(B, D)$. 
Proof. To compute the Hausdorff distance between two parallel line segments $\overline{A B}$ and $\overline{C D}$, we will consider two cases. In the first case, the projection of one line segment onto the line containing the other is contained in the other line segment. In the second case, the projection of one line segment is not contained in the other. Without loss of generality, we can assume that the two cases are as depicted in Figure 6 .
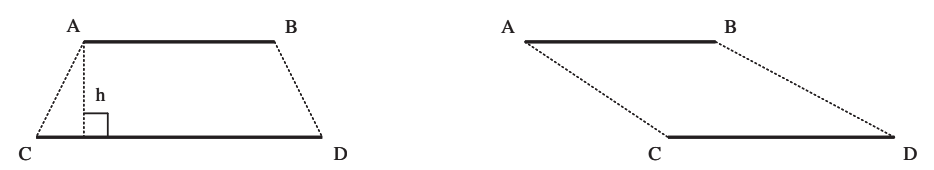

Figure 6. Case i (left): The projection of one line segment is contained in the other. Case ii (right): The projection of one line segment is not contained in the other.

Case i: Assume that the projection of $\overline{A B}$ onto the line containing $\overline{C D}$ is contained in $\overline{C D}$ (see Figure 5(left)). In this case,

$$
\begin{aligned}
d(\overline{A B}, \overline{C D}) & =\max \{d(x, \overline{C D}): x \in \overline{A B}\} \\
& =h \\
d(\overline{C D}, \overline{A B}) & =\max \{d(x, \overline{A B}): x \in \overline{C D}\} \\
& =d(A, C) \vee d(B, D)
\end{aligned}
$$

Since $h$ is always less than or equal to $|A C|$ and $|B D|, h(\overline{A B}, \overline{C D})=d(\overline{A B}, \overline{C D}) \vee$ $d(\overline{C D}, \overline{A B})=d(A, C) \vee d(B, D)$.

Case ii: Now assume that the projection of one line segment is not contained in the other line segment (see Figure 5(right)). Then

$$
\begin{aligned}
d(\overline{A B}, \overline{C D}) & =\max \{d(x, \overline{C D}): x \in \overline{A B}\} \\
& =d(A, C) \\
d(\overline{C D}, \overline{A B}) & =\max \{d(x, \overline{A B}): x \in \overline{C D}\} \\
& =d(B, D)
\end{aligned}
$$

Hence $h(\overline{A B}, \overline{C D})=d(A, C) \vee d(B, D)$ holds for the second case as well, and this completes the proof of the lemma.

Also of use in this paper is the following lemma which computes the Hausdorff distance between the two polygons that result from applying two consecutive $K_{2 k}$ maps to the turtle programs $T M_{2 k+2}$ and $\overline{T M}_{2 k+2}$.

Lemma 3.4.3. For $k \geq 3, h\left(K_{2 k}\left[T M_{2 k+2}\right], K_{2 k+2}\left[T M_{2 k+2}\right]\right)=\frac{\sqrt{3}}{2}\left(l_{k}+3\right)$ and $h\left(K_{2 k}\left[\overline{T M}_{2 k+2}\right], K_{2 k+2}\left[\overline{T M}_{2 k+2}\right]\right)=\frac{\sqrt{3}}{2} l_{k}$

Proof. As illustrated in Figure 7, $K_{2 k+2}\left[T M_{2 k+2}\right]$ is the single line segment $\overline{A E}$ while $K_{2 k}\left[T M_{2 k+2}\right]$ is the polygonal curve consisting of the four line segments: $\overline{A B}, \overline{B C}, \overline{C D}$, and $\overline{D E}$.

To compute $h\left(K_{2 k}\left[T M_{2 k+2}\right], K_{2 k+2}\left[T M_{2 k+2}\right]\right)$, we need to compute the two distances $d\left(K_{2 k}\left[T M_{2 k+2}\right], K_{2 k+2}\left[T M_{2 k+2}\right]\right)$ and $d\left(K_{2 k+2}\left[T M_{2 k+2}\right], K_{2 k}\left[T M_{2 k+2}\right]\right)$. 


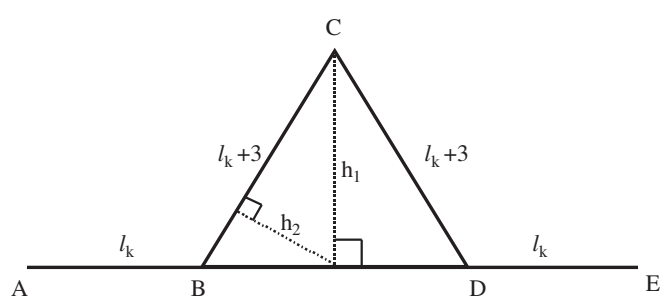

FiguRe 7 . The polygons $K_{2 k}\left[T M_{2 k+2}\right]$ and $K_{2 k+2}\left[T M_{2 k+2}\right]$

$$
\begin{aligned}
d\left(K_{2 k}\left[T M_{2 k+2}\right], K_{2 k+2}\left[T M_{2 k+2}\right]\right) & =\max \left\{d\left(x, K_{2 k+2}\left[T M_{2 k+2}\right]\right): x \in K_{2 k}\left[T M_{2 k+2}\right]\right\} \\
& =h_{1} \\
& =\sin (\pi / 3)\left(l_{k}+3\right)
\end{aligned}
$$

and

$$
\begin{aligned}
d\left(K_{2 k+2}\left[T M_{2 k+2}\right], K_{2 k}\left[T M_{2 k+2}\right]\right) & =\max \left\{d\left(x, K_{2 k}\left[T M_{2 k+2}\right]\right): x \in K_{2 k+2}\left[T M_{2 k+2}\right]\right\} \\
& =h_{2} \\
& =\frac{h_{1}}{2}
\end{aligned}
$$

We see then that $h\left(K_{2 k}\left[T M_{2 k+2}\right], K_{2 k+2}\left[T M_{2 k+2}\right]\right)=h_{1} \vee h_{2}=\sin (\pi / 3)\left(l_{k}+3\right)$. That is, $h\left(K_{2 k}\left[T M_{2 k+2}\right], K_{2 k+2}\left[T M_{2 k+2}\right]\right)=\frac{\sqrt{3}}{2}\left(l_{k}+3\right)$.

To compute $h\left(K_{2 k}\left[\overline{T M}_{2 k+2}\right], K_{2 k+2}\left[\overline{T M}_{2 k+2}\right]\right)$ we now refer to Figure 8. Much like the previous situation, $\left.K_{2 k+2}\left[\overline{T M}_{2 k+2}\right]\right)$ is the single line segment $\overline{A B}$ and $K_{2 k}\left[\overline{T M}_{2 k+2}\right]$ is the union of four line segments: $\overline{A B}, \overline{B C}, \overline{C D}$, and $\overline{D E}$.

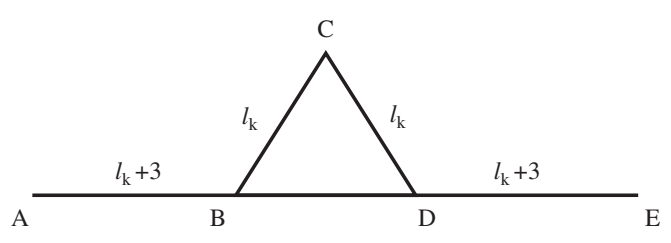

Figure 8 . The polygons $K_{2 k}\left[\overline{T M}_{2 k+2}\right]$ and $K_{2 k+2}\left[\overline{T M}_{2 k+2}\right]$

A computation similar to that of the previous case shows that

$$
h\left(K_{2 k}\left[\overline{T M}_{2 k+2}\right], K_{2 k+2}\left[\overline{T M}_{2 k+2}\right]\right)=\sin (\pi / 3) l_{k}=\frac{\sqrt{3}}{2} l_{k} .
$$

Finally, we list several useful properties of the Hausdorff metric. Some are included as standard exercises in textbooks (see, for example [2]), and the others easily checked.

Property 3.4.4. For all $A, B \in \mathcal{H}\left(\mathbb{R}^{2}\right)$ and $x \in \mathbb{R}^{2}, h(x+A, x+B)=h(A, B)$.

Property 3.4.5. For all $A, B \in \mathcal{H}\left(\mathbb{R}^{2}\right)$ and $\lambda \in \mathbb{R}, h(\lambda A, \lambda B)=\lambda h(A, B)$. 
Property 3.4.6. Let $R$ be a rotation matrix and $A, B \in \mathcal{H}\left(\mathbb{R}^{2}\right)$. Then $h(R \cdot A, R \cdot B)=$ $h(A, B)$.

Property 3.4.7. For all $A, B, C, D \in \mathcal{H}\left(\mathbb{R}^{2}\right), h(A \cup B, C \bigcup D) \leq h(A, C) \vee h(B, D)$.

As the next lemma shows, Property 3.4.4 and Property 3.4.6 together yield a fifth property involving the polygon maps $K_{2 k}$. This property will be useful in proving the convergence theorems.

Lemma 3.4.8. For any $w_{1}, w_{2}, w_{3} \in \Sigma_{\mathcal{R}}^{*}, h\left(g\left(w_{1}\right) \boxplus K_{2 k}\left[w_{2}\right], g\left(w_{1}\right) \boxplus K_{2 k}\left[w_{3}\right]\right)=$ $h\left(K_{2 k}\left[w_{2}\right], K_{2 k}\left[w_{3}\right]\right)$.

Proof. Let $w_{1}, w_{2}, w_{3} \in \Sigma_{\mathcal{R}}^{*}$, and let $\pi_{2} \circ \psi\left(w_{1}\right)=R$. Then

$$
\begin{aligned}
h\left(g\left(w_{1}\right) \boxplus K_{2 k}\left[w_{2}\right], g\left(w_{1}\right) \boxplus K_{2 k}\left[w_{3}\right]\right) & =h\left(g\left(w_{1}\right)+R K_{2 k}\left[w_{2}\right], g\left(w_{1}\right)+R K_{2 k}\left[w_{3}\right]\right) \\
& =h\left(R K_{2 k}\left[w_{2}\right], R K_{2 k}\left[w_{3}\right]\right) \\
& =h\left(K_{2 k}\left[w_{2}\right], K_{2 k}\left[w_{3}\right]\right)
\end{aligned}
$$

\section{Preliminary Convergence Results}

In this section, we examine the polygons $K_{2 k}\left[T M_{2 n}\right]$ more closely. In particular, we start by considering the difference between two consecutive polygon maps $K_{2 k}$ and $K_{2 k+2}$ applied to a given Thue-Morse program $T M_{2 n}$ (and scaled down by $S_{n}$ ). As the following result shows, when $k$ is large the difference is small.

Proposition 4.0.9. Let $h$ be the Hausdorff metric on $\mathcal{H}\left(\mathbb{R}^{2}\right)$ and $3 \leq k<n$. Then given any $\varepsilon>0$, there exists $N \in \mathbb{N}$ sufficiently large such that

$$
h\left(S_{n} K_{2 k}\left[T M_{2 n}\right], S_{n} K_{2 k+2}\left[T M_{2 n}\right]\right)<\varepsilon
$$

for all $n \geq N$.

Proof. Consider $T M_{2 n}=\prod_{i=1}^{4^{n-k-1}} v_{i}$, where $v_{i} \in\left\{T M_{2 k+2}, \overline{T M}_{2 k+2}\right\}$. Then $K_{2 k}\left[T M_{2 n}\right]=\bigcup_{i=1}^{4^{n-k-1}}\left(g\left(v_{1} v_{2} \ldots v_{i-1}\right) \boxplus K_{2 k}\left[v_{i}\right]\right)$. (Note: In the case where $i=1$, $g\left(v_{1} v_{2} \ldots v_{i-1}\right)=g(\varepsilon)$.)

By Property 3.4.5 we find that

$$
h\left(S_{n} K_{2 k}\left[T M_{2 n}\right], S_{n} K_{2 k+2}\left[T M_{2 n}\right]\right) \leq S_{n} \cdot h\left(K_{2 k}\left[T M_{2 n}\right], K_{2 k+2}\left[T M_{2 n}\right]\right)
$$

and because each of the polygons $K_{2 k}\left[T M_{2 n}\right]$ and $K_{2 k+2}\left[T M_{2 n}\right]$ is a union of edges of the form $g\left(v_{1} v_{2} \ldots v_{i-1}\right) \boxplus K_{2 k}\left[v_{i}\right]$ and $g\left(v_{1} v_{2} \ldots v_{i-1}\right) \boxplus K_{2 k+2}\left[v_{i}\right]$ respectively, we can apply Property 3.4.7 to show that the right-hand side of $(*)$ is bounded above by

$$
\left.S_{n} \cdot \max _{i=1}^{4^{n-k-1}}\left\{h\left(g\left(v_{1} v_{2} \ldots v_{i-1}\right) \boxplus K_{2 k}\left[v_{i}\right]\right), g\left(v_{1} v_{2} \ldots v_{i-1}\right) \boxplus K_{2 k+2}\left[v_{i}\right]\right)\right\} .
$$

Hence, by Lemma 3.4.8, we conclude that $h\left(K_{2 k}\left[T M_{2 n}\right], K_{2 k+2}\left[T M_{2 n}\right]\right) \leq S_{n}$. $\max _{i=1}^{4^{n-k-1}}\left\{h\left(K_{2 k}\left[v_{i}\right], K_{2 k+2}\left[v_{i}\right]\right)\right\}$, and since $v_{i} \in\left\{T M_{2 k+2}, \overline{T M}_{2 k+2}\right\}$ for all $i$, each $h\left(K_{2 k}\left[v_{i}\right], K_{2 k+2}\left[v_{i}\right]\right)$ is bounded above by the maximum of $h\left(K_{2 k}\left[T M_{2 k+2}\right]\right.$, $\left.K_{2 k+2}\left[T M_{2 k+2}\right]\right)$ and $h\left(K_{2 k}\left[\overline{T M}_{2 k+2}\right], K_{2 k+2}\left[\overline{T M}_{2 k+2}\right]\right)$. Applying Lemma 3.4.3 then yields

$$
\begin{aligned}
h\left(S_{n} K_{2 k}\left[T M_{2 n}\right], S_{n} K_{2 k+2}\left[T M_{2 n}\right]\right) & \leq \max \left\{S_{n} \sin (\pi / 3)\left(l_{k}+3\right), S_{n} \sin (\pi / 3) l_{k}\right\} \\
& \leq S_{n} \sin (\pi / 3)\left(l_{k}+3\right) \\
& \leq \frac{2}{3^{n-2}-1} \cdot \frac{3^{k-1}+3}{{ }^{2}} \cdot \frac{\sqrt{3}}{2} \\
& \leq \frac{3^{k-1}+3}{3^{n-2}-1} \cdot \frac{\sqrt{3}^{2}}{2}
\end{aligned}
$$


Since $k$ is fixed, it is easy to see now that we can make $\frac{3^{k-1}+3}{3^{n-2}-1} \cdot \frac{\sqrt{3}}{2}$ arbitrarily small by making $n$ sufficiently large.

The previous theorem still holds if $k=\frac{n}{2}$ for even $n$ or $k=\frac{n+1}{2}$ for odd $n$. When $n$ is even we find that $h\left(S_{n} K_{2 k}\left[T M_{2 n}\right], S_{n} K_{2 k+2}\left[T M_{2 n}\right]\right) \leq \frac{3^{\frac{n^{2}-1}{2}}+3}{3^{n-2}-1} \cdot \frac{\sqrt{3}}{2}$. The case for odd $n$ is similar. However, the theorem does not hold any more if $k=n-m$ for any finite $m$, because in this case we get $h\left(S_{n} K_{2 k}\left[T M_{2 n}\right], S_{n} K_{2 k+2}\left[T M_{2 n}\right]\right) \leq$ $\frac{3^{n-m-1}+3}{3^{n-2}-1} \cdot \frac{\sqrt{3}}{2} \leq 3^{1-m} \cdot \frac{\sqrt{3}}{2}$ which does not approach 0 with increasing $n$. This implies that $k$ can be chosen to be arbitrarily large because as $n$ approaches infinity, $\frac{n}{2}$ approaches infinity too. Nevertheless, $k$ cannot be arbitrarily close to $n$. Moreover, we can generalize the proposition for any two finite $K_{2 k}$ maps, not necessarily the consecutive ones.

Proposition 4.0.10. Let $h$ be the Hausdorff metric on $\mathcal{H}\left(\mathbb{R}^{2}\right)$ and $j, k \in \mathbb{N}$ satisfying $3 \leq j \leq k$. Then given any $\varepsilon>0$, there exists $N \in \mathbb{N}$ sufficiently large such that

$$
h\left(S_{n} K_{2 j}\left[T M_{2 n}\right], S_{n} K_{2 k}\left[T M_{2 n}\right]\right)<\varepsilon
$$

for all $n \geq N$.

Proof. The proof starts out similarly to that of the previous result. Suppose $T M_{2 n}=\prod_{i=1}^{4^{n-k}} v_{i}$ where $v_{i} \in\left\{T M_{2 k}, \overline{T M}_{2 k}\right\}$. Once again apply Properties 3.4.5, 3.4.7 and Lemma 3.4.8 to conclude

$$
h\left(S_{n} K_{2 j}\left[T M_{2 n}\right], S_{n} K_{2 k}\left[T M_{2 n}\right]\right) \leq S_{n} \cdot \max _{i=1}^{4^{n-k}}\left\{h\left(K_{2 j}\left[v_{i}\right], K_{2 k}\left[v_{i}\right]\right)\right\}
$$

Since $v_{i} \in\left\{T M_{2 k}, \overline{T M}_{2 k}\right\}, h\left(K_{2 j}\left[v_{i}\right], K_{2 k}\left[v_{i}\right]\right)$ is bounded above by

$$
\max \left\{h\left(K_{2 j}\left[T M_{2 k}\right], K_{2 k}\left[T M_{2 k}\right]\right), h\left(K_{2 j}\left[{\overline{T M_{2 k}}}\right], K_{2 k}\left[\overline{\left.T M_{2 k}\right]}\right)\right\} .\right.
$$

The triangle inequality then yields

$$
h\left(K_{2 j}\left[T M_{2 k}\right], K_{2 k}\left[T M_{2 k}\right]\right) \leq \sum_{m=j}^{k-1} h\left(K_{2 m}\left[T M_{2 k}\right], K_{2 m+2}\left[T M_{2 k}\right]\right)
$$

and

$$
h\left(K_{2 j}\left[\overline{T M}_{2 k}\right], K_{2 k}\left[{\left.\overline{T M_{2 k}}\right]}\right) \leq \sum_{m=j}^{k-1} h\left(K_{2 m}\left[\overline{T M}_{2 k}\right], K_{2 m+2}\left[\overline{T M}_{2 k}\right]\right) .\right.
$$

Next note that for each $j \leq m \leq k-1, T M_{2 k}$ and $\overline{T M}_{2 k}$ can both be rewritten as products: $T M_{2 k}=\prod_{i=1}^{4^{k-m-1}} w_{m, i}$ and $\overline{T M_{2 k}}=\prod_{i=1}^{4^{k-m-1}} \overline{w_{m, i}}$, where $w_{m, i} \in$ $\left\{T M_{2 m+2}, \overline{T M}_{2 m+2}\right\}$. Therefore $h\left(K_{2 m}\left[T M_{2 k}\right], K_{2 m+2}\left[T M_{2 k}\right]\right)$ and $h\left(K_{2 m}\left[\overline{T M}_{2 k}\right]\right.$, $\left.K_{2 m+2}\left[\overline{T M}_{2 k}\right]\right)$ are both bounded above by

$$
\max \left\{h\left(K_{2 m}\left[T M_{2 m+2}\right], K_{2 m+2}\left[T M_{2 m+2}\right]\right), h\left(K_{2 m}\left[\overline{T M}_{2 m+2}\right], K_{2 m+2}\left[\overline{T M}_{2 m+2}\right]\right)\right\} .
$$

We conclude that $h\left(K_{2 j}\left[T M_{2 n}\right], K_{2 k}\left[T M_{2 n}\right]\right)$ is bounded above by

$$
\left.\sum_{m=j}^{k-1} \max \left\{h\left(K_{2 m}\left[T M_{2 m+2}\right], K_{2 m+2}\left[T M_{2 m+2}\right]\right)\right), h\left(K_{2 m}\left[\overline{T M}_{2 m+2}\right], K_{2 m+2}\left[\overline{T M}_{2 m+2}\right]\right)\right\} .
$$


Finally, by Lemma $3.4 .3, h\left(K_{2 m}\left[T M_{2 m+2}\right], K_{2 m+2}\left[T M_{2 m+2}\right]\right)=\sin (\pi / 3)\left(l_{m}+3\right)$ and $h\left(K_{2 m}\left[\overline{T M}_{2 m+2}\right], K_{2 m+2}\left[\overline{T M}_{2 m+2}\right]\right)=\sin (\pi / 3) l_{m}$. Hence

$$
\begin{aligned}
h\left(S_{n} K_{2 j}\left[T M_{2 n}\right], S_{n} K_{2 k}\left[T M_{2 n}\right]\right) & \leq S_{n} \cdot h\left(K_{2 j}\left[T M_{2 n}\right], K_{2 k}\left[T M_{2 n}\right]\right) \\
& \leq S_{n} \cdot \sum_{m=j}^{k-1} \sin (\pi / 3)\left(l_{m}+3\right) \\
& \leq S_{n} \cdot(k-j) \sin (\pi / 3)\left(l_{k-1}+3\right) \\
& \leq \frac{3^{k-2}+3}{3^{n-2}-1} \cdot(k-j) \cdot \frac{\sqrt{3}}{2}
\end{aligned}
$$

Clearly, for finite $j$ and $k, h\left(S_{n} K_{2 j}\left[T M_{2 n}\right], S_{n} K_{2 k}\left[T M_{2 n}\right]\right)$ approaches 0 as $n$ approaches infinity.

Remark 4.0.11. The previous two propositions make up a key component to the proof of the convergence of the polygonal Thue-Morse curves $\left\{S_{n} K_{2 k}\left(T M_{2 n}\right)\right\}_{n=3}^{\infty}$. Furthermore, if $\left\{S_{n} K_{2 k}\left(T M_{2 n}\right)\right\}_{n=3}^{\infty}$ converges to $K(T M) \in \mathcal{H}\left(\mathbb{R}^{2}\right)$ in the Hausdorff metric, then the propositions tell us that the set $K(T M)$ does not depend on our choice of $k$. That is, we can use the polygon map $K_{2 k}: \Sigma_{\mathcal{R}}^{*} \rightarrow \mathcal{H}\left(\mathbb{R}^{2}\right)$ of our choice. This is a very powerful result.

We prove one final lemma before presenting our main results.

Lemma 4.0.12. For all $w_{1}, w_{2}, v_{1}, v_{2} \in \Sigma_{\mathcal{R}}^{*}$ satisfying $\pi_{2} \circ \psi\left(w_{1}\right)=\pi_{2} \circ \psi\left(v_{1}\right)$,

$$
d\left(g\left(w_{1}\right) \boxplus g\left(w_{2}\right), g\left(v_{1}\right) \boxplus g\left(v_{2}\right)\right) \leq d\left(g\left(w_{1}\right), g\left(v_{1}\right)\right)+d\left(g\left(w_{2}\right), g\left(v_{2}\right)\right)
$$

Proof. Suppose $\pi_{2} \circ \psi\left(w_{1}\right)=\pi_{2} \circ \psi\left(v_{1}\right)=R$. Then

$$
\begin{aligned}
& d\left(g\left(w_{1}\right) \boxplus g\left(w_{2}\right), g\left(v_{1}\right) \boxplus g\left(v_{2}\right)\right)=d\left(g\left(w_{1}\right)+R \cdot g\left(w_{2}\right), g\left(v_{1}\right)+R \cdot g\left(v_{2}\right)\right) \\
& =\left|\left(\overrightarrow{g\left(w_{1}\right)+R \cdot g\left(w_{2}\right)}\right)-\left(\overrightarrow{g\left(v_{1}\right)+R \cdot g\left(v_{2}\right)}\right)\right| \\
& \left.=\mid \overrightarrow{\left(g\left(w_{1}\right)\right.}-\overrightarrow{g\left(v_{1}\right)}\right)+\left(\overrightarrow{R \cdot g\left(w_{2}\right)}-\overrightarrow{R \cdot g\left(v_{2}\right)}\right) \mid \\
& \left.\leq \mid \overrightarrow{\left(g\left(w_{1}\right)\right.}-\overrightarrow{g\left(v_{1}\right)}\right)|+|\left(\overrightarrow{R \cdot g\left(w_{2}\right)}-\overrightarrow{R \cdot g\left(v_{2}\right)}\right) \mid \\
& \leq d\left(g\left(w_{1}\right), g\left(v_{1}\right)\right)+d\left(R \cdot g\left(w_{2}\right), R \cdot g\left(v_{2}\right)\right) \\
& \leq d\left(g\left(w_{1}\right), g\left(v_{1}\right)\right)+d\left(g\left(w_{2}\right), g\left(v_{2}\right)\right)
\end{aligned}
$$

\section{The Main Results}

Theorem 5.0.13. (Convergence Theorem I) For positive integer $n \geq 8$, let

$$
k_{n}= \begin{cases}\frac{n}{2} & \text { if } n \text { is even } \\ \frac{n+1}{2} & \text { if } n \text { is odd }\end{cases}
$$

Then the sequence of compact sets $\left\{S_{n} K_{2 k_{n}}\left[T M_{2 n}\right]\right\}_{n=8}^{\infty}$ converges in the Hausdorff metric.

Proof. In order to prove convergence, we show that the sequence is Cauchy. That is, we prove that for each $\epsilon>0$ there exists a natural number $N$ such that for all $n, m>N, h\left(S_{n} K_{2 k_{n}}\left[T M_{2 n}\right], S_{m} K_{2 k_{n}}\left[T M_{2 m}\right]\right)<\epsilon$. Without loss of generality, we will assume that $n \leq m$. Choosing $N_{1}, N_{2} \in \mathbb{N}$ to be such that $324 \cdot\left(\frac{2}{3}\right)^{N_{1}}<\frac{\epsilon}{2}$ and $2 \sqrt{3} \cdot\left(\frac{1}{3}\right)^{\frac{N_{2}}{4}}<\frac{\epsilon}{2}$, we let $N=\max \left\{N_{1}, N_{2}\right\}$. By the triangle inequality and the symmetry of $h$, we get that $h\left(S_{n} K_{2 k_{n}}\left[T M_{2 n}\right], S_{m} K_{2 k_{n}}\left[T M_{2 m}\right]\right)$ is bounded above by

$$
h\left(S_{n} K_{2 k_{n}}\left[T M_{2 n}\right], S_{m} K_{2 k_{m}}\left[T M_{2 m}\right]\right)+h\left(S_{m} K_{2 k_{m}}\left[T M_{2 m}\right], S_{m} K_{2 k_{n}}\left[T M_{2 m}\right]\right) .
$$


By triangle inequality again, the left hand side of the summand is bounded above by

$$
\sum_{i=n}^{m-1} h\left(S_{i} K_{2 k_{i}}\left[T M_{2 i}\right], S_{i+1} K_{2 k_{i}+2}\left[T M_{2 i+2}\right]\right) .
$$

We will show next that each term $h\left(S_{i} K_{2 k_{i}}\left[T M_{2 i}\right], S_{i+1} K_{2 k_{i}+2}\left[T M_{2 i+2}\right]\right)$ in the above sum is bounded above by $\frac{6 \cdot 2^{i}}{3^{i-2}-1}$. Then when $i \geq 3$ we have $\frac{1}{3^{i-2}-1}<\frac{2}{3^{i-2}}$, and therefore, $h\left(S_{i} K_{2 k_{i}}\left[T M_{2 i}\right], S_{i+1} K_{2 k_{i}+2}\left[T M_{2 i+2}\right]\right)<108 \cdot\left(\frac{2}{3}\right)^{i}$.

To prove $h\left(S_{i} K_{2 k_{i}}\left[T M_{2 i}\right], S_{i+1} K_{2 k_{i}+2}\left[T M_{2 i+2}\right]\right)<\frac{6 \cdot 2^{i}}{3^{i-2}-1}$, suppose $T M_{2 i}=$ $\prod_{j=1}^{4^{i-k_{i}}} v_{j}$ and $T M_{2 i+2}=\prod_{j=1}^{4^{i-k_{i}}} w_{j}$ where $v_{j} \in\left\{T M_{2 k_{i}}, \overline{T M}_{2 k_{i}}\right\}$ and $w_{j} \in\left\{T M_{2 k_{i}+2}, \overline{T M}_{2 k_{i}+2}\right\}$. Then $K_{2 k_{i}}\left[T M_{2 i}\right]=\bigcup_{j=1}^{4^{i-k_{i}}}\left(g\left(v_{1} v_{2} \ldots v_{j-1}\right) \boxplus K_{2 k_{i}}\left[v_{j}\right]\right)$ and $K_{2 k_{i}+2}\left[T M_{2 i+2}\right]=\bigcup_{j=1}^{4^{i-k_{i}}}\left(g\left(w_{1} w_{2} \ldots w_{j-1}\right) \boxplus\right.$ $\left.K_{2 k_{i}+2}\left[w_{j}\right]\right)$. By Property 3.4.7 $h\left(S_{i} K_{2 k_{i}}\left[T M_{2 i}\right], S_{i+1} K_{2 k_{i}+2}\left[T M_{2 i+2}\right]\right)$ is equal to

$$
\max _{j=1}^{4^{i-k_{i}}}\left\{h\left(S_{i}\left(g\left(v_{1} v_{2} \ldots v_{j-1}\right) \boxplus K_{2 k_{i}}\left[v_{j}\right]\right), S_{i+1}\left(g\left(w_{1} w_{2} \ldots w_{j-1}\right) \boxplus K_{2 k_{i}+2}\left[w_{j}\right]\right)\right)\right\} .
$$

Next note that $K_{2 k_{i}}$ and $K_{2 k_{i}+2}$ are two consecutive polygon maps and $w_{j}=$ $\sigma^{2}\left(v_{j}\right)$. Hence $g\left(v_{1} v_{2} \ldots v_{j-1}\right) \boxplus K_{2 k_{i}}\left[v_{j}\right]$ and $g\left(w_{1} w_{2} \ldots w_{j-1}\right) \boxplus K_{2 k_{i}+2}\left[w_{j}\right]$ are parallel line segments, and by Lemma 3.4 .2 we can compute $h\left(S_{i} K_{2 k_{i}}\left[T M_{2 i}\right], S_{i+1} K_{2 k_{i}+2}\left[T M_{2 i+2}\right]\right)$ by computing the distances between the endpoints of edges. In particular, $h\left(S_{i} K_{2 k_{i}}\left[T M_{2 i}\right], S_{i+1} K_{2 k_{i}+2}\left[T M_{2 i+2}\right]\right)$ equals

$$
\max _{j=1}^{4^{i-k_{i}}}\left\{d\left(S_{i}\left(g\left(v_{1} v_{2} \ldots v_{j-1}\right) \boxplus g\left(v_{j}\right)\right), S_{i+1}\left(g\left(w_{1} w_{2} \ldots w_{j-1}\right) \boxplus g\left(w_{j}\right)\right)\right)\right\} .
$$

Since $\pi_{2} \circ \psi\left(v_{j}\right)=\pi_{2} \circ \psi\left(w_{j}\right)=R_{\theta}^{2}$ for all $j$ and $\pi_{2} \circ \psi$ is a homomorphism, $\pi_{2} \circ \psi\left(v_{1} v_{2} \ldots v_{j-1}\right)=\pi_{2} \circ \psi\left(w_{1} w_{2} \ldots w_{j-1}\right)$. By Lemma 4.0.12, we conclude then that $h\left(S_{i} K_{2 k_{i}}\left[T M_{2 i}\right], S_{i+1} K_{2 k_{i}+2}\left[T M_{2 i+2}\right]\right)$ is bounded above by

$$
\max _{j=1}^{4^{i-k_{i}}}\left\{d\left(S_{i} g\left(v_{1} v_{2} \ldots v_{j-1}\right), S_{i+1} g\left(w_{1} w_{2} \ldots w_{j-1}\right)+d\left(S_{i} g\left(v_{j}\right), S_{i+1} g\left(w_{j}\right)\right)\right\} .\right.
$$

Moreover, recall that $g: \Sigma_{\mathcal{R}}^{*} \rightarrow \mathbb{R}^{2}$ is a homomorphism, so $g\left(v_{1} v_{2} \ldots v_{j-1}\right)=g\left(v_{1}\right) \boxplus$ $g\left(v_{2}\right) \boxplus \ldots \boxplus g\left(v_{j-1}\right)$. Hence we can continue to apply Lemma 4.0.12:

$$
\begin{aligned}
& h\left(S_{i} K_{2 k_{i}}\right.\left.\left.T M_{2 i}\right], S_{i+1} K_{2 k_{i}+2}\left[T M_{2 i+2}\right]\right) \\
& \leq \max _{j=1}^{4^{i-k_{i}}}\left\{d\left(S_{i}\left(g\left(v_{1} v_{2} \ldots v_{j-2}\right) \boxplus g\left(v_{j-1}\right)\right), S_{i+1}\left(g\left(w_{1} w_{2} \ldots w_{j-2}\right) \boxplus g\left(w_{j-1}\right)\right)\right)\right.\left.\quad d\left(S_{i} g\left(v_{j}\right), S_{i+1} g\left(w_{j}\right)\right)\right\} \\
& \leq \max _{j=1}^{4^{i-k_{i}}} \quad\left\{d\left(S_{i} g\left(v_{1} v_{2} \ldots v_{j-2}\right), S_{i+1} g\left(w_{1} w_{2} \ldots w_{j-2}\right)\right)+d\left(S_{i} g\left(v_{j-1}\right), S_{i+1} g\left(w_{j-1}\right)\right)\right. \\
&\left.\quad+d\left(S_{i} g\left(v_{j}\right), S_{i+1} g\left(w_{j}\right)\right)\right\} \\
& \leq \max _{j=1}^{4^{i-k_{i}}}\left\{\sum_{l=1}^{j} d\left(S_{i} g\left(v_{l}\right), S_{i+1} g\left(w_{l}\right)\right)\right\} \\
& \leq \sum_{l=1}^{4^{i-k_{i}}}\left\{d\left(S_{i} g\left(v_{l}\right), S_{i+1} g\left(w_{l}\right)\right)\right\} .
\end{aligned}
$$

Therefore, it suffices now to find an upper bound for $d\left(S_{i} g\left(v_{l}\right), S_{i+1} g\left(w_{l}\right)\right)$. We consider two cases:

Case i: $d\left(S_{i} g\left(v_{l}\right), S_{i+1} g\left(w_{l}\right)\right)=d\left(S_{i} g\left(T M_{2 k_{i}}\right), S_{i+1} g\left(T M_{2 k_{i}+2}\right)\right)$. In this case, we employ Proposition 3.2.2 to compute:

$$
\begin{aligned}
d\left(S_{i} g\left(T M_{2 k_{i}}\right), S_{i+1} g\left(T M_{2 k_{i}+2}\right)\right) & =\left|\overrightarrow{S_{i} g\left(T M_{2 k_{i}}\right)}-\overrightarrow{S_{i+1} g\left(T M_{2 k_{i}+2}\right)}\right| \\
& =\left|\frac{2}{3^{i-2}-1} \cdot \frac{3-3^{k_{i}-1}}{2} \cdot R_{\theta} \cdot \overrightarrow{v_{0}}-\frac{2}{3^{i-1}-1} \cdot \frac{3-3^{k_{i}}}{2} \cdot R_{\theta} \cdot \overrightarrow{v_{0}}\right| \\
& =\left|R_{\theta} \cdot \overrightarrow{v_{0}}\right| \cdot\left|\frac{2}{3^{i-2}-1} \cdot \frac{3-3^{k_{i}-1}}{2}-\frac{2}{3^{i-1}-1} \cdot \frac{3-3^{k_{i}}}{2} \cdot\right| \\
& =\frac{2 \cdot\left(3^{i-1}-3^{k_{i}-1}\right)}{\left(3^{i-2}-1\right)\left(3^{i-1}-1\right)},
\end{aligned}
$$


and since $k_{i} \geq 3$, we have

$$
\frac{2 \cdot\left(3^{i-1}-3^{k_{i}-1}\right)}{\left(3^{i-2}-1\right)\left(3^{i-1}-1\right)} \leq \frac{2 \cdot\left(3^{i-1}-3^{0}\right)}{\left(3^{i-2}-1\right)\left(3^{i-1}-1\right)} \leq \frac{2}{3^{i-2}-1} .
$$

Case ii: $d\left(S_{i} g\left(v_{l}\right), S_{i+1} g\left(w_{l}\right)\right)=d\left(S_{i} g\left(\overline{T M}_{2 k_{i}}\right), S_{i+1} g\left(\overline{T M}_{2 k_{i}+2}\right)\right)$. We handle the second case in a similar way. Once again, apply Proposition 3.2.2 to compute:

$$
\begin{aligned}
d\left(S_{i} g\left(\overline{T M}_{2 k_{i}}\right), S_{i+1} g\left(\overline{T M}_{2 k_{i}+2}\right)\right) & =\left|\overrightarrow{S_{i} g\left(\overline{T M}_{2 k_{i}}\right)}-\overrightarrow{S_{i+1} g\left(\overline{T M}_{2 k_{i}+2}\right)}\right| \\
& =\left|\frac{2}{3^{i-2}-1} \cdot \frac{3+3^{k_{i}-1}}{2} \cdot R_{\theta} \cdot \hat{v}_{0}-\frac{2}{3^{i-1}-1} \cdot \frac{3+3^{k_{i}}}{2} \cdot R_{\theta} \cdot \hat{v}_{0}\right| \\
& =\left|R_{\theta} \cdot \overrightarrow{v_{0}}\right| \cdot\left|\frac{2}{3^{i-2}-1} \cdot \frac{3+3^{k_{i}-1}}{2}-\frac{2}{3^{i-1}-1} \cdot \frac{3+3^{k_{i}}}{2} \cdot\right| \\
& =\frac{2 \cdot\left(3^{i-1}+3^{k_{i}-1}\right)}{\left(3^{i-2}-1\right)\left(3^{i-1}-1\right)} .
\end{aligned}
$$

Since $k_{i}<i$, we have

$$
\frac{2 \cdot\left(3^{i-1}+3^{k_{i}-1}\right)}{\left(3^{i-2}-1\right)\left(3^{i-1}-1\right)} \leq \frac{2 \cdot\left(3^{i-1}+3^{i-1}\right)}{\left(3^{i-2}-1\right)\left(3^{i-1}-1\right)} \leq \frac{4 \cdot\left(3^{i-1}\right)}{\left(3^{i-2}-1\right)\left(3^{i-1}-1\right)},
$$

and for $i \geq 2, \frac{3^{i-1}}{3^{i-1}-1} \leq \frac{3}{2}$. Hence, for $i \geq 2$

$$
\frac{2 \cdot\left(3^{i-1}+3^{k_{i}-1}\right)}{\left(3^{i-2}-1\right)\left(3^{i-1}-1\right)} \leq \frac{6}{\left(3^{i-2}-1\right)}
$$

and $\frac{6}{\left(3^{i-2}-1\right)}$ is an upper bound.

Taking the larger of the two upper bounds, we see then that $\frac{6}{\left(3^{i-2}-1\right)}$ serves as an upper bound for $d\left(S_{i} g\left(v_{l}\right), S_{i+1} g\left(w_{l}\right)\right)$ in both cases, and we can return to our computation...

$$
\begin{aligned}
h\left(S_{i} K_{2 k_{i}}\left[T M_{2 i}\right], S_{i+1} K_{2 k_{i}+2}\left[T M_{2 i+2}\right]\right) & \leq \sum_{l=1}^{4^{i-k_{i}}} d\left(S_{i} g\left(v_{l}\right), S_{i+1} g\left(w_{l}\right)\right) \\
& \leq 4^{i-\bar{k}_{i}} \cdot \frac{6}{\left(3^{i-2}-1\right)}
\end{aligned}
$$

If $i$ is even and $k_{i}=\frac{i}{2}$, then $4^{i-k_{i}} \cdot \frac{6}{\left(3^{i-2}-1\right)}=4^{\frac{i}{2}} \cdot \frac{6}{\left(3^{i-2}-1\right)}=\frac{6 \cdot 2^{i}}{\left(3^{i-2}-1\right)}$, and therefore

$$
h\left(S_{i} K_{2 k_{i}}\left[T M_{2 i}\right], S_{i+1} K_{2 k_{i}+2}\left[T M_{2 i+2}\right]\right) \leq \frac{6 \cdot 2^{i}}{\left(3^{i-2}-1\right)} .
$$

The case where $i$ is odd is similar, and hence $h\left(S_{i} K_{2 k_{i}}\left[T M_{2 i}\right], S_{i+1} K_{2 k_{i}+2}\left[T M_{2 i+2}\right]\right)<$ $108 \cdot\left(\frac{2}{3}\right)^{i}$. Thus

$$
\begin{aligned}
\sum_{i=n}^{m-1} h\left(S_{i} K_{2 k_{i}}\left[T M_{2 i}\right], S_{i+1} K_{2 k_{i}+2}\left[T M_{2 i+2}\right]\right) & <\sum_{i=n}^{m-1} 108 \cdot\left(\frac{2}{3}\right)^{i} \\
& =324 \cdot\left[\left(\frac{2}{3}\right)^{n}-\left(\frac{2}{3}\right)^{m}\right] \\
& <324 \cdot\left(\frac{2}{3}\right)^{n} \\
& <324 \cdot\left(\frac{2}{3}\right)^{N} \\
& <\frac{\epsilon}{2}
\end{aligned}
$$

Now consider the right hand term of the summand. By Proposition 4.0.10, $h\left(S_{m} K_{2 k_{m}}\left[T M_{2 m}\right], S_{m} K_{2 k_{n}}\left[T M_{2 m}\right]\right)$ is bounded above by $\frac{3^{k_{m}-2}+3}{3^{m}-1} \cdot\left(k_{m}-k_{n}\right) \cdot \frac{\sqrt{3}}{2}$. If $m, n$ are both even, the bound is equal to $\frac{3^{\frac{m}{2}-2}+3}{3^{m}-1} \cdot \frac{m-n}{2} \cdot \frac{\sqrt{3}}{2}$. Other cases are similar, and because $m<3^{\frac{m}{4}}(m \geq 8)$, for $m$ sufficiently large we have 


$$
\begin{aligned}
\frac{3^{\frac{m}{2}-2}+3}{3^{m}-1} \cdot \frac{m-n}{2} \cdot \frac{\sqrt{3}}{2} & <\frac{3^{\frac{m}{2}-2}+3}{3^{m}-1} \cdot m \cdot \frac{\sqrt{3}}{2} \\
& <\frac{3^{\frac{m}{2}}+3}{3^{m}-1} \cdot 3^{\frac{m}{4}} \cdot \frac{\sqrt{3}}{2} \\
& <\frac{2 \cdot 3^{\frac{3 m}{4}}}{3^{m}-1} \cdot \frac{\sqrt{3}}{2} \\
& <\frac{4 \cdot 3^{\frac{3 m}{4}}}{3^{m}} \cdot \frac{\sqrt{3}}{2} \\
& =2 \sqrt{3} \cdot\left(\frac{1}{3}\right)^{\frac{m}{4}} \\
& <2 \sqrt{3} \cdot\left(\frac{1}{3}\right)^{\frac{N}{4}} \\
& <\frac{\epsilon}{2}
\end{aligned}
$$

Therefore, we found a natural number $N$ such that for all $n, m>N$,

$$
\begin{aligned}
& h\left(S_{n} K_{2 k_{n}}\left[T M_{2 n}\right], S_{m} K_{2 k_{n}}\left[T M_{2 m}\right]\right) \\
\leq & h\left(S_{n} K_{2 k_{n}}\left[T M_{2 n}\right], S_{m} K_{2 k_{m}}\left[T M_{2 m}\right]\right)+h\left(S_{m} K_{2 k_{m}}\left[T M_{2 m}\right], S_{m} K_{2 k_{n}}\left[T M_{2 m}\right]\right) \\
< & \frac{\epsilon}{2}+\frac{\epsilon}{2}
\end{aligned}
$$

We conclude that the sequence $\left\{S_{n} K_{2 k_{n}}\left[T M_{2 n}\right]\right\}_{n=8}^{\infty}$ converges in the Hausdorff metric, and this completes the proof of the theorem.

Since $\left(\mathcal{H}\left(\mathbb{R}^{2}\right), h(\cdot, \cdot)\right)$ is a complete metric space [2], we can conclude further that $\left\{S_{n} K_{2 k_{n}}\left[T M_{2 n}\right]\right\}_{n=5}^{\infty}$ converges to a compact set. As the next theorem will show, this compact set is precisely the Koch snowflake $\mathcal{K}$ (as defined in Example 3.3.2).

Theorem 5.0.14. (Convergence Theorem II) For positive integer $n \geq 5$, let

$$
k_{n}= \begin{cases}\frac{n}{2} & \text { if } n \text { is even } \\ \frac{n+1}{2} & \text { if } n \text { is odd }\end{cases}
$$

Then the sequence $\left\{S_{n} K_{2 k_{n}}\left[T M_{2 n}\right]\right\}_{n=5}^{\infty}$ converges to $\mathcal{K}$ in the Hausdorff metric.

Proof. We prove the convergence by showing that $\mathcal{H}\left(S_{n} K_{2 k_{n}}\left[T M_{2 n}\right], \frac{1}{3^{n-k_{n}}} \mathcal{K}_{2 n-2 k_{n}}\right)$ approaches 0 as $n$ approaches infinity. Consider $T M_{2 n}=\prod_{i=1}^{4^{n-k_{n}}} v_{i}$, where $v_{i} \in$ $\left\{T M_{2 k_{n}}, \overline{T M}_{2 k_{n}}\right\}$ and $K S_{2 n-2 k_{n}}=\prod_{i=1}^{4^{n-k_{n}}} y_{i}$, where $y_{i} \in\left\{L^{4} F^{3} L^{4}, L F^{3} L\right\}$. Then $K_{2 k_{n}}\left[T M_{2 n}\right]=\bigcup_{i=1}^{4^{n-k_{n}}}\left(g\left(v_{1} v_{2} \ldots v_{i-1}\right) \boxplus K_{2 k_{n}}\left[v_{i}\right]\right)$ and $\mathcal{K}_{2 n-2 k_{n}}=\bigcup_{i=1}^{4^{n-k_{n}}}\left(g\left(y_{1} y_{2} \ldots y_{i-1}\right)\right.$ $\left.\boxplus K_{k s}\left[y_{i}\right]\right)$ (For the definition of $K_{k s}$ map, see Example 3.3.2). Since $\pi_{2} \circ \Phi\left(v_{i}\right)=$ $\pi_{2} \circ \Phi\left(y_{i}\right)=R_{\theta}^{2}$ for all $i$, the corresponding edges of $K_{2 k_{n}}\left[T M_{2 n}\right]$ and $\mathcal{K}_{2 n-2 k_{n}}$ (that is, $v_{i}$ and $\left.y_{i}\right)$ are parallel line segments. Hence, similar to the proof of Theorem 5.0.13, we apply Property 3.4.7, Lemma 3.4.2, and Lemma 4.0.12 (multiple times) to conclude that

$$
\begin{aligned}
& h\left(S_{n} K_{2 k_{n}}\left[T M_{2 n}\right], \frac{1}{3^{n-k_{n}}} \mathcal{K}_{2 n-2 k_{n}}\right) \\
& \leq \max _{i=1}^{4^{n-k_{n}}}\left\{h\left(S_{n}\left(g\left(v_{1} v_{2} \ldots v_{i-1}\right) \boxplus K_{2 k_{n}}\left[v_{i}\right]\right), \frac{1}{3^{n-k_{n}}}\left(g\left(y_{1} y_{2} \ldots y_{i-1}\right) \boxplus K_{k s}\left[y_{i}\right]\right)\right)\right\}
\end{aligned}
$$




$$
\begin{aligned}
& \leq \max _{i=1}^{4^{n-k_{n}}}\left\{h\left(S_{n}\left(g\left(v_{1} v_{2} \ldots v_{i-1}\right) \boxplus g\left(v_{i}\right)\right), \frac{1}{3^{n-k_{n}}}\left(g\left(y_{1} y_{2} \ldots y_{i-1}\right) \boxplus g\left(y_{i}\right)\right)\right)\right\} \\
& \leq \max _{i=1}^{4^{n-k_{n}}}\left\{\sum_{j=1}^{i} d\left(S_{n} g\left[v_{j}\right], \frac{1}{3^{n-k_{n}}} g\left[y_{j}\right]\right)\right\}
\end{aligned}
$$

Hence it suffices to compute $d\left(S_{n} K_{2 k_{n}}\left[v_{j}\right], \frac{1}{3^{n-k_{n}}} K_{k s}\left[y_{j}\right]\right)$. Once again, there are two cases to consider.

Case i: If $v_{j}=T M_{2 k_{n}}$ and $y_{j}=L^{4} F^{3} L^{4}$, then

$$
\begin{aligned}
d\left(S_{n} g\left(v_{j}\right), \frac{1}{3^{n-k_{n}}} g\left(y_{j}\right)\right) & =d\left(S_{n} g\left(T M_{2 k_{n}}\right), \frac{1}{3^{n-k_{n}}} g\left(L^{4} F^{3} L^{4}\right)\right) \\
& =\left|\overrightarrow{S_{n} g\left(T M_{2 k_{n}}\right)}-\frac{1}{3^{n-k_{n}}} g\left(L^{4} F^{3} L^{4}\right)\right| \\
& =\left|\frac{2}{3^{n-2}-1} \cdot \frac{3-3^{k_{n}-1}}{2} \cdot R_{\theta} \cdot \hat{v}_{0}-\frac{1}{3^{n-k_{n}}} \cdot(-3) \cdot R_{\theta} \cdot \hat{v}_{0}\right| \\
& =\left|R_{\theta} \cdot \hat{v}_{0}\right| \cdot\left|\frac{2}{3^{n-2}-1} \cdot \frac{3-3^{k_{n}-1}}{2}+\frac{1}{3^{n-k_{n}-1}}\right| \\
& =\frac{3\left(3^{n-k_{n}}-1\right)}{3^{n-k_{n} \cdot\left(3^{n-2}-1\right)}} \\
& \leq \frac{3}{\left(3^{n-2}-1\right)}
\end{aligned}
$$

Case ii: In the second case, $v_{j}=\overline{T M}_{2 k_{n}}$ and $y_{j}=L F^{3} L$. Then

$$
\begin{aligned}
d\left(S_{n} g\left(v_{j}\right), \frac{1}{3^{n-k_{n}}} g\left(y_{j}\right)\right) & =d\left(S_{n} g\left(\overline{T M}_{2 k_{n}}\right), \frac{1}{3^{n-k_{n}}} g\left(L F^{3} L\right)\right) \\
& =\left|\overrightarrow{S_{n} g\left(\overline{T M}_{2 k_{n}}\right)}-\frac{1}{3^{n-k_{n}}} g\left(L F^{3} L\right)\right| \\
& =\left|\frac{2}{3^{n-2}-1} \cdot \frac{3+3^{k_{n}-1^{2}}}{2} \cdot R_{\theta} \cdot \hat{v}_{0}-\frac{1}{3^{n-k_{n}}} \cdot 3 \cdot R_{\theta} \cdot \hat{v}_{0}\right| \\
& =\left|R_{\theta} \cdot \hat{v}_{0}\right| \cdot\left|\frac{2}{3^{n-2}-1} \cdot \frac{3+3^{k_{n}-1}}{2}-\frac{1}{3^{n-k_{n}-1}}\right| \\
& =\frac{3\left(3^{n-k_{n}}+1\right)}{3^{n-k_{n} \cdot\left(3^{n-2}-1\right)}} \\
& \leq \frac{3\left(3^{n-k_{n}}+3^{n-k_{n}}\right)}{3^{n-k_{n} \cdot\left(3^{n-2}-1\right)}} \\
& \leq \frac{6}{\left(3^{n-2}-1\right)}
\end{aligned}
$$

In either case, $d\left(S_{n} g\left(v_{j}\right), \frac{1}{3^{n-k_{n}}} g\left(y_{j}\right)\right) \leq \frac{6}{\left(3^{n-2}-1\right)}$, and therefore

$$
\begin{aligned}
h\left(S_{n} K_{2 k_{n}}\left[T M_{2 n}\right], \frac{1}{3^{n-k_{n}}} \mathcal{K}_{2 n-2 k_{n}}\right) & \leq \max _{i=1}^{4^{n-k_{n}}}\left\{\sum_{j=1}^{i} d\left(S_{n} g\left[v_{j}\right], \frac{1}{3^{n-k_{n}}} g\left[y_{j}\right]\right)\right\} \\
& \leq \sum_{j=1}^{4^{n-k_{n}}} d\left(S_{n} g\left[v_{j}\right], \frac{1}{3^{n-k_{n}}} g\left[y_{j}\right]\right) \\
& \leq 4^{n-k_{n}} \cdot \frac{6}{\left(3^{n-2}-1\right)}
\end{aligned}
$$

If $n$ is even, then $k_{n}=\frac{n}{2}$, and $h\left(S_{n} K_{2 k_{n}}\left[T M_{2 n}\right], \frac{1}{3^{n-k_{n}}} \mathcal{K}_{2 n-2 k_{n}}\right) \leq \frac{6 \cdot 2^{n}}{\left(3^{n-2}-1\right)}$. Clearly, we can make $\frac{6 \cdot 2^{n}}{\left(3^{n-2}-1\right)}$ arbitrarily small by increasing $n$, and the case where $n$ is odd is similar. We conclude then that $\left\{S_{n} K_{2 k_{n}}\left[T M_{2 n}\right]\right\}$ and $\left\{\mathcal{K}_{2 n-2 k_{n}}\right\}$ converge to the same compact set in the Hausdorff metric. That is, $\left\{S_{n} K_{2 k_{n}}\left[T M_{2 n}\right]\right\}$ converges to $\mathcal{K}$.

Hence, by using sufficiently coarse polygon maps, we see that the Thue-Morse sequence $\left\{T M_{2 n}\right\}_{n \geq 3}$ does indeed encode the Koch snowflake.

\section{A Generalization}

In previous sections of this paper, we have focused primarily on the Thue-Morse turtle sequence. At this point, we will broaden our focus somewhat by examining what we consider to be generalized Thue-Morse turtle sequences. To be more concise, if $w, w^{\prime} \in \Sigma_{\mathcal{R}}^{*}$ and $\sigma$ is the same substitution map defined in the introduction, then $\sigma^{2}$ defines the sequence of turtle programs: $\left\{\sigma^{2 n}(w)\right\}_{n \geq 1}$. A generalized Thue-Morse sequence is defined to be the $\operatorname{limit}_{n \rightarrow \infty} \sigma^{2 n}(w)$. A natural question to ask next is: 
Which $w, w^{\prime} \in \Sigma_{\mathcal{R}}^{*}$ generate turtle programs $\left\{\sigma^{2 n}(w)\right\}_{n \geq k}$ that encode turtle trajectories converging to the Koch snowflake?

To answer this question, we first observe that if $\left\{\sigma^{2 n}(w)\right\}_{n \geq k}$ encodes the Koch snowflake, then the replacements $w \rightarrow \sigma^{2}(w)$ and $w^{\prime} \rightarrow \sigma^{2}\left(w^{\prime}\right)$ should reflect the replacement rule used to define the Koch snowflake. Referring to Figure 9 below, we see that this occurs when $w$ and $w^{\prime}$ satisfy the following three properties:

(1) $\overrightarrow{g(w)}$ and $\overrightarrow{g\left(w^{2}\right)}$ form two sides of an equilateral triangle, meaning that $|\overrightarrow{g(w)}|=\left|\overrightarrow{g\left(w^{2}\right)}\right|$. The similar statement must hold true for $w^{\prime}$.

(2) $\overrightarrow{g\left(w w^{\prime} w^{\prime}\right)}=c_{1} \overrightarrow{g(w)}$ for some real number $c_{1}>1$

(3) $\overrightarrow{g\left(w w^{\prime} w^{\prime} w\right)}=c_{2} \overrightarrow{g(w)}$ for some real number $c_{2}>c_{1}>1$

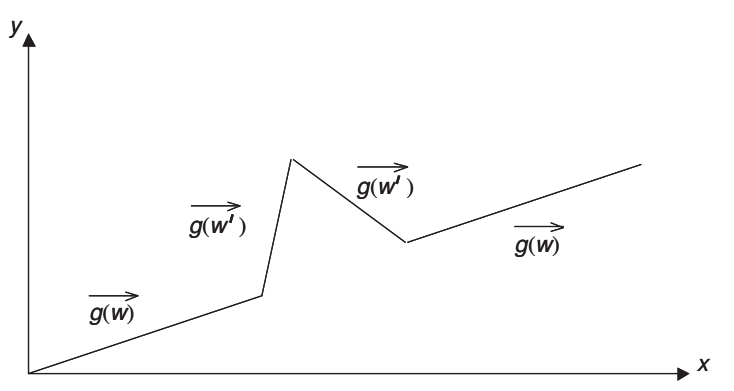

FIGURE 9. The replacement structure corresponding to $\sigma^{2}(w)$

It is not difficult to characterize the words $w$ and $w^{\prime}$ that satisfy these properties. As it turns out, $w$ and $w^{\prime}$ will have to be such that $|w|_{L}=\left|w^{\prime}\right|_{L} \bmod 6$ and $\overrightarrow{g(w)}$ and $\overrightarrow{g\left(w^{\prime}\right)}$ lay in the same line but in the opposite direction. We will prove two lemmas before establishing this result.

Lemma 6.0.15. If $w \in \Sigma_{\mathcal{R}}^{*}$ with $|w|_{L}=m$, then $|\overrightarrow{g(w)}|=\left|\overrightarrow{g\left(w^{2}\right)}\right|$ if and only if $m= \pm 2 \bmod 6$.

Proof. Assume $\pi_{1} \circ \psi(w)=M$ and $|w|_{L}=m$. Then $\pi_{1} \circ \psi\left(w^{2}\right)=M+R_{\theta}^{m} M=$ $\left(I+R_{\theta}^{m}\right) M$. Observe that $|\overrightarrow{g(w)}|=\left|\pi_{1} \circ \psi(w) \cdot \widehat{v}_{0}\right|=\sqrt{\operatorname{det} M}$ and $\left|g\left(w^{2}\right)\right|=$ $\sqrt{\operatorname{det}\left(I+R_{\theta}^{m}\right)} \sqrt{\operatorname{det} M}$. Hence $|\overrightarrow{g(w)}|=\left|\overrightarrow{g\left(w^{2}\right)}\right|$ if and only if $\operatorname{det}\left(I+R_{\theta}^{m}\right)=1$. A simple calculation then shows that $\operatorname{det}\left(I+R_{\theta}^{m}\right)=1$ precisely when $m= \pm 2$ $\bmod 6$.

Hence property (1) forces $|w|_{L}$ and $\left|w^{\prime}\right|_{L}$ to be congruent to either 2 or 4 modulo 6. Assuming that properties (2) and (3) hold as well, we can conclude further that $|w|_{L}=\left|w^{\prime}\right|_{L} \bmod 6$.

Lemma 6.0.16. If $w, w^{\prime} \in \Sigma_{\mathcal{R}}^{*}$ with $|w|_{L}=m$ and $\left|w^{\prime}\right|_{L}=n$ and $\left\{w, w^{\prime}\right\}$ satisfy properties (1), (2), and (3) above, then $m=n= \pm 2 \bmod 6$.

Proof. If $w$ and $w^{\prime}$ satisfy properties (1), (2), and (3), and $|w|_{L}=m$ and $\left|w^{\prime}\right|_{L}=n$, then

$$
\begin{aligned}
\overrightarrow{g\left(w w^{\prime} w^{\prime} w\right)} & =\overrightarrow{g\left(w w^{\prime} w^{\prime}\right)}+R_{\theta}^{m+2 n} \overrightarrow{g(w)} \\
& =c_{1} \overrightarrow{g(w)}+R_{\theta}^{m+2 n} \overrightarrow{g(w)}
\end{aligned}
$$


Since $\overrightarrow{g\left(w w^{\prime} w^{\prime} w\right)}=c_{2} \overrightarrow{g(w)}$, we see then that $R_{\theta}^{m+2 n} \overrightarrow{g(w)}=\left(c_{2}-c_{1}\right) \overrightarrow{g(w)}$, and hence $R_{\theta}^{m+2 n}=\left(c_{2}-c_{1}\right) I$ is a scalar matrix. Since $c_{2}>c_{1}$, we conclude that $R_{\theta}^{m+2 n}$ is a positive scalar matrix, and therefore, $R_{\theta}^{m+2 n}=I$. Equivalently, $m+2 n=0 \bmod 6$, and since $m$ and $n$ are congruent to $\pm 2 \bmod 6$ (by Lemma 6.0.15), we conclude that $m=n \bmod 6$.

Note that the above proof actually shows that $c_{2}$ depends on $c_{1}$; in fact, $c_{2}=$ $1+c_{1}$. We are now ready to complete our characterization of $w$ and $w^{\prime}$.

Theorem 6.0.17. Two words $w$ and $w^{\prime}$ in $\Sigma_{\mathcal{R}}^{*}$ satisfy the three properties:

(1) $|\overrightarrow{g(w)}|=\left|\overrightarrow{g\left(w^{2}\right)}\right|$ and $\left|\overrightarrow{g\left(w^{\prime}\right)}\right|=\left|\overrightarrow{g\left(\left(w^{\prime}\right)^{2}\right)}\right|$.

(2) $\overrightarrow{g\left(w w^{\prime} w^{\prime}\right)}=c_{1} \overrightarrow{g(w)}$ for some real number $c_{1}>1$

(3) $\overrightarrow{g\left(w w^{\prime} w^{\prime} w\right)}=c_{2} \overrightarrow{g(w)}$ for some real number $c_{2}>c_{1}>1$

if and only if $|w|_{L}=\left|w^{\prime}\right|_{L} \bmod 6$, and $\overrightarrow{g(w)}$ and $\overrightarrow{g\left(w^{\prime}\right)}$ lay in the same line but in the opposite direction.

Proof. Assume $w$ and $w^{\prime}$ in $\Sigma_{\mathcal{R}}^{*}$ satisfy properties (1), (2), and (3). If $|w|_{L}=m$ and $\left|w^{\prime}\right|_{L}=n$, then $\psi(w)=\left(M, R_{\theta}^{m}\right)$ and $\psi\left(w^{\prime}\right)=\left(M^{\prime}, R_{\theta}^{n}\right)$ for some $M, M^{\prime} \in M_{2}(\mathbb{R})$, and

$$
\begin{aligned}
\overrightarrow{g\left(w w^{\prime} w^{\prime}\right)} & =\left(M+R_{\theta}^{m} M^{\prime}+R_{\theta}^{m+n} M^{\prime}\right) \cdot \widehat{v}_{0} \\
& =\left(M+\left(R_{\theta}^{m}+R_{\theta}^{m+n}\right) M^{\prime}\right) \cdot \widehat{v}_{0} \\
& =\overrightarrow{g(w)}+\left(R_{\theta}^{m}+R_{\theta}^{m+n}\right) \overrightarrow{g\left(w^{\prime}\right)} .
\end{aligned}
$$

Since $\overrightarrow{g\left(w w^{\prime} w^{\prime}\right)}=c_{1} \overrightarrow{g(w)},\left(c_{1}-1\right) \overrightarrow{g(w)}=\left(R_{\theta}^{m}+R_{\theta}^{m+n}\right) \overrightarrow{g\left(w^{\prime}\right)}$, and by the previous lemma, $m=n= \pm 2 \bmod 6$. Hence $R_{\theta}^{m}+R_{\theta}^{m+n}=R_{\theta}^{m}+R_{\theta}^{2 m}=-I$, and $\left(c_{1}-1\right) \overrightarrow{g(w)}=-\overrightarrow{g\left(w^{\prime}\right)}$. Therefore, $\overrightarrow{g(w)}=-\frac{1}{\left(c_{1}-1\right)} \overrightarrow{g\left(w^{\prime}\right)}$, and since $c_{1}>1$, we conclude that $\overrightarrow{g(w)}$ and $\overrightarrow{g\left(w^{\prime}\right)}$ lay in the same line but in the opposite direction.

Conversely, assume that $\overrightarrow{g(w)}=-d \overrightarrow{g\left(w^{\prime}\right)}$ for some $d>0$, and let $m=|w|_{L}=$ $\left|w^{\prime}\right|_{L}= \pm 2 \bmod 6$. By Lemma 6.0.15, we already know that $w$ and $w^{\prime}$ satisfy the first property. Next compute:

$$
\begin{aligned}
\overrightarrow{g\left(w w^{\prime} w^{\prime}\right)} & =\overrightarrow{g(w)}+R_{\theta}^{m} \overrightarrow{g\left(w^{\prime}\right)}+R_{\theta}^{2 m} \overrightarrow{g\left(w^{\prime}\right)} \\
& =I-d\left(R_{\theta}^{m}+R_{\theta}^{2 m}\right) \overrightarrow{g(w)} \\
& =(1+d) \overrightarrow{g(w)}
\end{aligned}
$$

Since $d>0,1+d>1$, and we see that $w$ and $w^{\prime}$ satisfy the second property. For the third property, compute:

$$
\begin{aligned}
\overrightarrow{g\left(w w^{\prime} w^{\prime} w\right)} & =\overrightarrow{g\left(w w^{\prime} w^{\prime}\right)}+R_{\theta}^{3 m} \overrightarrow{g(w)} \\
& =(1+d) \overrightarrow{g(w)}+\overrightarrow{g(w)} \\
& =(2+d) \overrightarrow{g(w)}
\end{aligned}
$$

Clearly, $2+d>1+d>1$, and hence $w$ and $w^{\prime}$ satisfy property (3) as well.

In light of this characterization of $w$ and $w^{\prime}$, it is interesting to revisit the situation where $w=T M_{2 n}$ and $w^{\prime}=\overline{T M}_{2 n}$, with $n \geq 3$. Indeed, one can check that $\left|T M_{2 n}\right|_{L}=\left|\overline{T M}_{2 n}\right|_{L}=2^{2 n-1}=2 \bmod 6$, and Proposition 3.2.2 ensures that $\overrightarrow{g\left(T M_{2 n}\right)}$ and $\overrightarrow{g\left(\overline{T M}_{2 n}\right)}$ have opposite directions when $n \geq 3$. Hence 
$\left\{T M_{2 n}, \overline{T M}_{2 n}\right\}$ satisfy our characterization - as we would expect. As the next theorem illustrates, we could have established the same results without dealing with an arbitrary $n$. In particular, proving that $T M_{6}$ satisfies the two criteria would have been enough.

Theorem 6.0.18. If $w$ and $w^{\prime}$ are two words in $\sum_{\mathcal{R}}^{*}$ satisfying the properties

(1) $|w|_{L}=\left|w^{\prime}\right|_{L}= \pm 2 \bmod 6$

(2) $\overrightarrow{g(w)}$ and $\overrightarrow{g\left(w^{\prime}\right)}$ lay along the same line but in the opposite direction, then for all $n \geq 0, \sigma^{2 n}(w)$ and $\sigma^{2 n}\left(w^{\prime}\right)$ satisfy properties (1) and (2) as well.

Proof. Assuming $\left\{w, w^{\prime}\right\}$ and $\left\{\sigma^{2 n}(w), \sigma^{2 n}\left(w^{\prime}\right)\right\}$ satisfy the two properties, consider the pair $\left\{\sigma^{2 n+2}(w), \sigma^{2 n+2}\left(w^{\prime}\right)\right\}$. The proof that $\left\{\sigma^{2 n+2}(w), \sigma^{2 n+2}\left(w^{\prime}\right)\right\}$ satisfies property (1) is similar to the proof of the first part of Lemma 3.2.1. If $\left|\sigma^{2 n}(w)\right|_{L}=$ $\left|\sigma^{2 n}\left(w^{\prime}\right)\right|_{L}=k= \pm 2 \bmod 6$, then $\pi_{2} \circ \psi\left(\sigma^{2 n}(w)\right)=\pi_{2} \circ \psi\left(\sigma^{2 n}\left(w^{\prime}\right)\right)=R_{\theta}^{k}$, and

$$
\begin{aligned}
\pi_{2} \circ \psi\left(\sigma^{2 n+2}(w)\right) & =\pi_{2} \circ \psi\left(\sigma^{2 n}(w) \sigma^{2 n}\left(w^{\prime}\right) \sigma^{2 n}\left(w^{\prime}\right) \sigma^{2 n}(w)\right) \\
& =\left(R_{\theta}^{k}\right)^{4} \\
& =R_{\theta}^{k}
\end{aligned}
$$

Similarly, $\pi_{2} \circ \psi\left(\sigma^{2 n+2}\left(w^{\prime}\right)\right)=R_{\theta}^{k}$, and we see that $\left|\sigma^{2 n+2}(w)\right|_{L}=\left|\sigma^{2 n+2}\left(w^{\prime}\right)\right|_{L}=$ $k= \pm 2 \bmod 6$ as well.

Now assume that $\pi_{1} \circ \psi\left(\sigma^{2 n}(w)\right)=M$ and $\pi_{1} \circ \psi\left(\sigma^{2 n}\left(w^{\prime}\right)\right)=M^{\prime}$, and assume that $\left\{\sigma^{2 n}(w), \sigma^{2 n}\left(w^{\prime}\right)\right\}$ satisfies property (2). Then $M=c M^{\prime}$ for some negative real number $c$, and by Lemma 3.2.1,

$$
g\left(\sigma^{2 n+2}(w)\right)=\left(2 M-M^{\prime}\right) \cdot \widehat{v}_{0}=(2 c-1) M^{\prime} \cdot \widehat{v}_{0}
$$

and

$$
g\left(\sigma^{2 n+2}\left(w^{\prime}\right)\right)=\left(2 M^{\prime}-M\right) \cdot \widehat{v}_{0}=(2-c) M^{\prime} \cdot \widehat{v}_{0}
$$

Therefore, $g\left(\sigma^{2 n+2}(w)\right)=\frac{2 c-1}{2-c} \cdot g\left(\sigma^{2 n+2}\left(w^{\prime}\right)\right)$. Since $c$ is negative, $\frac{2 c-1}{2-c}$ is negative too. We see then that $\overrightarrow{g\left(\sigma^{2 n+2}(w)\right)}$ and $\overrightarrow{g\left(\sigma^{2 n+2}\left(w^{\prime}\right)\right)}$ are parallel, but point in the opposite direction. That is, $\left\{\sigma^{2 n+2}(w), \sigma^{2 n+2}\left(w^{\prime}\right)\right\}$ satisfies property (2).

Certainly, the formulation of the Koch snowflake given in section 2 (with $w=$ $L F^{3} L$ and $w^{\prime}=L^{4} F^{3} L^{4}$ ) satisfies the characterization described above. To illustrate the results of Theorems 6.0.17 and 6.0.18, we close this section with a couple of more interesting examples.

Example 6.0.19. If $w=L F L^{5} F L F L$ and $w^{\prime}=L^{3} F L F^{2} L^{4}$, then $|w|_{L}=\left|w^{\prime}\right|_{L}=$ $2 \bmod 6$ and $\overrightarrow{g(w)}=-\overrightarrow{g\left(w^{\prime}\right)}$. Below are the trajectories $K_{\text {turt }}\left[\sigma^{2 n}(w)\right]$ for $n=3$.

In the previous example, $w$ and $w^{\prime}$ represented edges of the same length. As the next example illustrates, this is by no means necessary. In fact, || $\overrightarrow{g(w)}|-| \overrightarrow{g\left(w^{\prime}\right)}||$ can be as large as we like.

Example 6.0.20. If $w=L F L^{5} F L F L$ and $w^{\prime}=L^{3} F L F^{2} L^{5} F L F^{2} L^{4}$, then $|w|_{L}=$ $\left|w^{\prime}\right|_{L}=2 \bmod 6$ and $\overrightarrow{g(w)}=-2 \overrightarrow{g\left(w^{\prime}\right)}$. Below is the trajectory $K_{\text {turt }}\left[\sigma^{2 n}(w)\right]$ for $n=4$. 


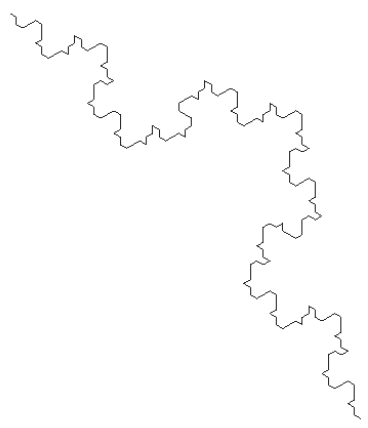

Figure 10. The polygon $K_{\text {turt }}\left[\sigma^{6}(w)\right]$, where $w=L F L^{5} F L F L$ and $w^{\prime}=L^{3} F L F^{2} L^{4}$

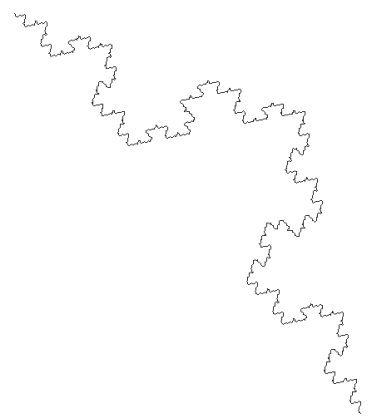

Figure 11 . The polygon $K_{\text {turt }}\left[\sigma^{8}(w)\right]$, where $w=L F L^{5} F L F L$ and $w^{\prime}=L^{3} F L F^{2} L^{5} F L F^{2} L^{4}$

\section{Conclusion}

In the previous section, we found that there are, in fact, many pairs $\left\{w, w^{\prime}\right\}$ that encode the Koch snowflake under iteration of the substitution map $\sigma^{2}$. One simply needs to define $w$ and $w^{\prime}$ to be such that they satisfy properties (1) and (2) of Theorem 6.0.18. It is worth noting, however, that in the original Thue-Morse sequence, $w^{\prime}$ is closely linked to $w$. In particular, $w^{\prime}=\bar{w}$ meaning that $w^{\prime}$ is obtained from $w$ by changing all $F$ 's to $L$ 's and all $L$ 's to $F$ 's. This leads us to one final question:

Is it possible to find a $w \in \Sigma_{R}^{*}$ not of the form $T M_{2 n}$ or $\overline{T M}_{2 n}$ such that the pair $\left\{w, w^{\prime}\right\}=\{w, \bar{w}\}$ generates turtle programs $\left\{\sigma^{2 n}(w)\right\}_{n \geq k}$ that encode turtle trajectories converging to the Koch snowflake?

This, it appears, is a much more difficult question to answer. We have not been able to find such a $w$, nor have we proved that one does not exist. What is clear, however, is that such a $w$ would have to meet a much more stringent set of criteria. For example, by Lemma 6.0 .15 , it is not hard to see that $w$ would have to satisfy $|w|_{L}=|w|_{F}= \pm 2 \bmod 6$, implying that $|w|=|\bar{w}|= \pm 2 \bmod 6$. If the ThueMorse turtle programs $T M_{2 n}$ and $\overline{T M}_{2 n}$ were the only words of the form $\{w, \bar{w}\}$ 
encoding the Koch snowflake, then this would establish an even tighter link between the Thue-Morse sequence and the Koch snowflake.

\section{ACKNOWLEDGMENT}

The second author would like to thank the Department of Mathematics at the University of Colorado - Boulder, and David Grant, Robert Tubbs, and Lynn Walling in particular, for the support they provided during the writing of this paper.

\section{REFERENCES}

1. H. Abelson and A.A. diSessa, Turtle geometry, MIT Press Series in Artificial Intelligence, MIT Press, Massachusetts, 1981.

2. M. Barnsley, Fractals everywhere, Academic Press, Inc., 1988.

3. J. Berstel, Axel Thue's papers on repetitions in words: a translation, Publications du LACIM, Université du Québec à Montréal, No. 20,1995.

4. F. M. Dekking, Recurrent sets, Advances in Math. 44 (1982), 78-104.

5. J. Holdener and A. Wagaman, A classification of periodic turtle sequences, International Journal of Mathematics and Mathematical Sciences 2003 (2003), no. 34, 2193-2201.

6. M. Lothaire, Combinatorics on words, Addison-Wesley, 1983.

7. A. Luca and S. Varricchio, Some combinatorial properties of the Thue-Morse sequence and a problem in semigroups, Theoretical Computer Science 63 (1989), 333-348.

8. M. Morse, Recurrent geodesics on a surface of negative curvature, Transactions Amer. Math. Soc. 22 (1921), 84-100.

9. M. Morse and G. Hedlund, Unending chess, symbolic dynamics and a problem in semigroups, Duke Math. J. 11 (1944), 1-7.

10. J.J. Pansiot, The Morse sequence and iterated morphisms, Inform. Process Lett. 12 (1981), 68-70.

11. P. Prusinkiewicz and A. Lindenmayer, The algorithmic beauty of plants, Springer-Verlag, 1990.

12. A. Thue, Über die gegenseitige lage gleicher teile gewisser zeichenreihen, Kra. Vidensk. Selsk. Skrifter. I. Mat.-Nat. Kl., Christiana 1912, Nr. 10.

13. H. von Koch, Une méthode géométrique élémentaire pour l'étude de certaines questions de la theorie des courbes planes, Acta Math. 30 (1906), 145-174.

Department of Mathematics, Kenyon College, Gambier, OH 43022

E-mail address: maj@kenyon.edu and holdenerj@kenyon.edu 